Research Article

\title{
European Option Pricing under Wishart Processes
}

\author{
Raphael Naryongo (D), ${ }^{1}$ Philip Ngare, ${ }^{2}$ and Anthony Waititu ${ }^{1}$ \\ ${ }^{1}$ Department of Statistics and Actuarial Science, Jomo Kenyatta University, Nairobi, Kenya \\ ${ }^{2}$ School of Mathematics, University of Nairobi, Nairobi, Kenya \\ Correspondence should be addressed to Raphael Naryongo; raphael.naryongo@students.jkuat.ac.ke
}

Received 10 May 2021; Revised 18 June 2021; Accepted 22 June 2021; Published 12 July 2021

Academic Editor: Efthymios G. Tsionas

Copyright (c) 2021 Raphael Naryongo et al. This is an open access article distributed under the Creative Commons Attribution License, which permits unrestricted use, distribution, and reproduction in any medium, provided the original work is properly cited.

\begin{abstract}
This study deals with a single risky asset pricing model whose volatility is described by Wishart affine processes. This multifactor model with two dependency matrices describing the correlation between the asset dynamic and Wishart processes makes it more flexible enough to fit the market data for short or long maturities. The aim of the study is to derive and solve the call option pricing problem under the double Wishart stochastic volatility model. The Fourier transform techniques combined with perturbation methods are employed in order to price the European call options. The numerical illustrations on pricing predictions show similar behavior of price movements under the double Wishart model with respect to the market price.
\end{abstract}

\section{Introduction}

The limitation of the standard Black and Scholes [1] model of not accounting for the fact that implied volatility of derivative products varies by strike and maturity; this makes it less flexible to reproduce certain market conditions observed on derivative prices. This caused the introduction of the Heston [2] model, which has been remarkably famous and heavily applied in financial markets due to its flexibility, financial interpretation of parameters, and analytical tractability property of the model since it belongs to the class of the affine process. This helps it to obtain the call price of a European option by inverting the Fourier transform and forming a closed-form solution of the log-price characteristic function.

However, despite the Heston model popularity, the existing literature documented the limitation of the model. Da Fonseca et al. [3], Christoffersen et al. [4], Ahdida and Alfonsi [5], Kang et al. [6], La Bua and Marazzina [7], and Gourieroux [8] pointed out that the major deficiency of the model does not generate the realistic term structure of the volatility smiles. The Heston model provides too flat implied volatility surface to attain reality. Yet, the implied volatility curve generally has steep slope, and it is convex in short maturity and tends to be linear for long maturity.

The literature recommends that the problem of not capturing such stylized facts can be solved by generalizing the Heston model using two approaches: by adding jump in the stock dynamic or volatility and investigating the multifactor nature of the implied volatility as emphasized in the studies by Benabid et al. [9], Da Fonseca et al. [3], La Bua and Marazzina [7], and Kang and Kang [10]. It is well understood that the multifactor approach is capable of handling the pricing problem of derivative products and volatility skew. This usher in the application of the stochastic matrix defined process, that is, the Wishart multidimensional stochastic volatility model. The Wishart process was introduced in Bru [11] and is defined as a positive semidefinite matrix-valued generalization of the square root process. The square root process is a Bessel process which is a generalization of the multiple chi-squared distribution $[12,13]$. The Wishart stochastic volatility model found its application in finance by Gourieroux and Sufana [14], and it has been applied in financial markets because of its nature of matrix specification which makes it flexible. 
The aim of this study is to price European option under two Wishart variance processes, through generalization of the Heston model into the multifactor nature of volatility modeling with two dependence matrices for single asset, that is, the asset dynamics depends on two Wishart volatility processes (double Wishart volatility processes). Following Kang et al. [6] and Benabid et al. [9], the matrix specification of the Wishart model makes it flexible enough to describe the market prices. The numerical illustrations show that price predictions under the double Wishart model have a similar behavior like the market prices.

The European call option pricing problem is handled through transform methods of Duffie et al. [15] and fast Fourier transform by Carr and Madan [16] combined with perturbation techniques of Benabid et al. [9] and Fouque et al. [17], in order to obtain the European call option price formula.

The study is structured as follows: Section 2 presents the definition of the Wishart process, and we introduce the Wishart stochastic volatility model and double Wishart model, correlation structures, and the infinitesimal generator of the Bi-Wishart model. Section 3 provides the solution for European call option pricing problem through Fourier transform methods combined with perturbation techniques. Section 4 presents the numerical illustrations to compare the price predictions under the double Wishart model with the market prices. Section 5 provides conclusion and further research work, together with technical proofs in the Appendix.

\section{Wishart Process}

Definition 1. Let $W_{t}, t \geq 0$ under the probability measure $\mathbb{Q}$ be a $n \times n$ matrix-valued Brownian motion. The Wishart matrix process $\Sigma$ satisfies the following equation:

$$
\mathrm{d} \Sigma_{t}=\left(\beta Q Q^{T}+M \Sigma_{t}+M \Sigma_{t}^{T}\right) \mathrm{d} t+\sqrt{\Sigma_{t}} \mathrm{~d} W_{t} Q+Q^{T} \mathrm{~d} W_{t}^{T} \sqrt{\Sigma_{t}},
$$

where $Q \in G L_{n}(\mathbb{R})$ is the invertible matrix, $M \in M_{n}$ is the nonpositive matrix, and $\Sigma_{0} \in \widetilde{S}_{n}^{+}$is the nonnegative symmetric matrix, while $\beta$ is a real parameter. The condition $\beta>(n-1)$ is considered to ensure existence and uniqueness of the $\Sigma_{t} \in \widetilde{S}_{n}^{+}$solution for equation (1), and Eigen values of the solution are nonnegative for all $t \geq 0$ a.s $\Sigma_{t} \in \widetilde{S}_{n}^{+}$. Like in the study by Benabid et al. [9], the probability measure $\mathbb{Q}$ corresponds to a risk-neutral measure (Appendix: change of the probability measure).

2.1. Wishart Volatility Model in the Stock Market. In Da Fonseca et al. [3], under the risk-neutral probability measure, the risky asset price dynamic and its quadratic variation are follows:

$$
\begin{aligned}
\frac{\mathrm{d} S(t)}{S(t)} & =r \mathrm{~d} t+\operatorname{Tr}\left[\sqrt{\Sigma_{t}} \mathrm{~d} Z(t)\right], \\
S_{0} & =s, \\
\mathrm{~d} \Sigma_{t} & =\left(\beta Q Q^{T}+M \Sigma_{t}+M \Sigma_{t}^{T}\right) \mathrm{d} t+\sqrt{\Sigma_{t}} \mathrm{~d} W_{t} Q+Q^{T} \mathrm{~d} W_{t}^{T} \sqrt{\Sigma_{t}}, \\
\Sigma_{0} & =\Sigma,
\end{aligned}
$$

where $r$ denotes risk-less interest rate, $\operatorname{Tr}$ is the trace, $Z \in M_{n}$ is the Brownian matrix, and $\Sigma_{t}$ is in a set of symmetric $n \times n$ positive-definite matrices. We observe that asset volatility is a trace of $\Sigma_{t}$ matrix, which is a multidimensional process with $M, Q \in M_{n}$, and $W_{t} \in M_{n}$ is the Brownian matrix.

In the study by Bru [11], the Wishart process provides a matrix analogue of the square root mean-reverting process and $M$ is considered negative to ensure positivity and meanreverting property of the volatility with parameter $\beta>n-1$ for uniqueness and existence of the solution.

2.2. Correlation Structure. The Brownian matrices $W_{t}$ and $Z_{t}$ are correlated, in such a way that it gives a constant correlated matrix $R \in M_{n}$, like in the study by Da Fonseca et al. [3], which describes the correlation structure, in such a way that $Z_{t}$ can be presented as follows:

$$
Z_{t}:=W_{t} R^{T}+B_{t} \sqrt{\square-R R^{T}},
$$

where $\llbracket$ denotes the identity matrix, $T$ is the transpose, and $B_{t}$ is an independent matrix Brownian motion from $W_{t}$. The correlation structure is a Brownian motion (Appendix).

2.3. Bivariate Wishart Stochastic Processes in Stock Market. In this section, we present a proposed novel model, the multifactor model with two Wishart variance processes or double Wishart stochastic volatility model with two dependence matrices. The model takes two volatility components which is the trace of Wishart whose diagonal components will be the controlling factors for the dynamics of volatilities.

Under arbitrage-free financial market and probability measure, we consider risky asset dynamic as follows:

$$
\begin{aligned}
\frac{\mathrm{d} S(t)}{S(t)} & =r \mathrm{~d} t+\operatorname{Tr}\left[\sqrt{\Sigma_{t}} \mathrm{~d} Z_{t}+\sqrt{\bar{\Sigma}_{t}} \mathrm{~d} \bar{Z}_{t}\right], \\
S_{0} & =s, \\
\mathrm{~d} \Sigma_{t} & =\left(\beta Q Q^{T}+M \Sigma_{t}+\Sigma_{t} M^{T}\right) \mathrm{d} t+\sqrt{\Sigma_{t}} \mathrm{~d} W_{t} Q+Q^{T} \mathrm{~d} W_{t}^{T} \sqrt{\Sigma_{t},} \\
\Sigma_{0} & =\Sigma, \\
\mathrm{d} \bar{\Sigma}_{t} & =\left(\bar{\beta}_{\overline{\mathrm{QQ}}}^{T}+\overline{M \Sigma}_{t}+\bar{\Sigma}_{t} \bar{M}^{T}\right) \mathrm{d} t+\sqrt{\bar{\Sigma}_{t}} \mathrm{~d} \bar{W}_{t} \overline{\mathrm{Q}}+\overline{\mathrm{Q}}^{T} \mathrm{~d} \bar{W}_{t}^{T} \sqrt{\bar{\Sigma}_{t}}, \\
\bar{\Sigma}_{0} & =\bar{\Sigma},
\end{aligned}
$$


where $\beta$ and $\bar{\beta}$ are the real parameters, such that $\beta, \bar{\beta}>n-1$, $\mathrm{Q}, \bar{Q}, M, \bar{M} \in M_{n}, \quad Q \quad$ is the invertible matrix, and $W_{t}, \bar{W}_{t} \in M_{n}$ are the matrices and Brownian motions, also $Z_{t}, \bar{Z}_{t} \in M_{n}$.

Lemma 1. The correlations between the Brownian matrices of the stock price dynamic and the Brownian matrices of the Wishart processes in equation (4) are given by

$$
\begin{gathered}
\rho_{t}=\frac{\operatorname{Tr}\left(R^{T} Q \Sigma_{t}\right)}{\sqrt{\operatorname{Tr}\left(\Sigma_{t}\right)} \sqrt{\operatorname{Tr}\left(Q^{T} Q \Sigma_{t}\right)}}, \\
\overline{\rho_{t}}=\frac{\operatorname{Tr}\left(\bar{R}^{T} \overline{\left.Q \Sigma_{t}\right)}\right.}{\sqrt{\operatorname{Tr}\left(\bar{\Sigma}_{t}\right)} \sqrt{\operatorname{Tr}\left(\bar{Q}^{T} \bar{Q}_{t}\right)}} .
\end{gathered}
$$

Proof. We derive the correlations as follows:

$$
\begin{aligned}
\frac{\mathrm{d} S_{t}}{S_{t}} & =r \mathrm{~d} t+\operatorname{Tr}\left[\sqrt{\Sigma_{t}} \mathrm{~d} Z_{t}+\sqrt{\bar{\Sigma}_{t}} \mathrm{~d} \bar{Z}_{t}\right] \\
& =r \mathrm{~d} t+\sqrt{\operatorname{Tr}\left(\Sigma_{t}\right)} \frac{\operatorname{Tr}\left(\sqrt{\Sigma_{t}} \mathrm{~d} Z_{t}\right)}{\sqrt{\operatorname{Tr}\left(\Sigma_{t}\right)}}+\sqrt{\operatorname{Tr}\left(\bar{\Sigma}_{t}\right)} \frac{\operatorname{Tr}\left(\sqrt{\bar{\Sigma}_{t}} \mathrm{~d} \bar{Z}_{t}\right)}{\sqrt{\operatorname{Tr}\left(\bar{\Sigma}_{t}\right)}} \\
& =r \mathrm{~d} t+\sqrt{\operatorname{Tr}\left(\Sigma_{t}\right)} \mathrm{d} X_{t}+\sqrt{\operatorname{Tr}\left(\bar{\Sigma}_{t}\right)} \mathrm{d} \bar{X}_{t},
\end{aligned}
$$

where $X_{t}$ and $\bar{X}_{t}$ are the standard Brownian motions (Appendix), and also taking the trace of the Wishart volatility dynamics (4), we have

$$
\begin{aligned}
& \mathrm{d} \operatorname{Tr}\left(\Sigma_{t}\right)=\left(\left(\beta \operatorname{Tr}\left(Q^{T} Q\right)\right)+2 \operatorname{Tr}\left(M \Sigma_{t}\right)\right) \mathrm{d} t+2 \operatorname{Tr}\left(Q \mathrm{~d} W_{t} \sqrt{\Sigma_{t}}\right), \\
& \mathrm{d} \operatorname{Tr}\left(\bar{\Sigma}_{t}\right)=\left(\left(\bar{\beta} \operatorname{Tr}\left(\bar{Q}^{T} \bar{Q}\right)\right)+2 \operatorname{Tr}\left(\overline{M \Sigma}_{t}\right)\right) \mathrm{d} t+2 \operatorname{Tr}\left(\bar{Q} \mathrm{~d} \bar{W}_{t} \sqrt{\bar{\Sigma}_{t}}\right) .
\end{aligned}
$$

These processes can still be written in the following form:

$$
\begin{aligned}
\mathrm{d} \operatorname{Tr}\left(\Sigma_{t}\right)= & \left(\left(\beta \operatorname{Tr}\left(Q^{T} Q\right)\right)+2 \operatorname{Tr}\left(M \Sigma_{t}\right)\right) \mathrm{d} t \\
& +2 \sqrt{\operatorname{Tr}\left(Q^{T} Q \Sigma_{t}\right)} \frac{\operatorname{Tr}\left(Q \mathrm{~d} W_{t} \sqrt{\Sigma_{t}}\right)}{\sqrt{\operatorname{Tr}\left(Q^{T} Q \Sigma_{t}\right)}}, \\
\mathrm{d} \operatorname{Tr}\left(\bar{\Sigma}_{t}\right)= & \left(\left(\bar{\beta} \operatorname{Tr}\left(\overline{\mathrm{Q}}^{T} \overline{\mathrm{Q}}\right)\right)+2 \operatorname{Tr}\left(\overline{M \Sigma_{t}}\right)\right) \mathrm{d} t \\
& +2 \sqrt{\operatorname{Tr}\left(\overline{\mathrm{Q}}^{T} \overline{\mathrm{Q}}_{t}\right)} \frac{\operatorname{Tr}\left(\overline{\mathrm{Q}}^{\mathrm{d}} \bar{W}_{t} \sqrt{\bar{\Sigma}_{t}}\right)}{\sqrt{\operatorname{Tr}\left(\overline{\mathrm{Q}}^{T} \overline{\mathrm{Q}}_{t}\right)}} .
\end{aligned}
$$

$$
\begin{aligned}
& \mathrm{d} \operatorname{Tr}\left(\Sigma_{t}\right)=\left(\left(\beta \operatorname{Tr}\left(Q^{T} Q\right)\right)+2 \operatorname{Tr}\left(M \Sigma_{t}\right)\right) \mathrm{d} t+2 \sqrt{\operatorname{Tr}\left(Q^{T} Q \Sigma_{t}\right)} \mathrm{d} \xi_{t}, \\
& \mathrm{~d} \operatorname{Tr}\left(\bar{\Sigma}_{t}\right)=\left(\left(\bar{\beta} \operatorname{Tr}\left(\bar{Q}^{T} \bar{Q}\right)\right)+2 \operatorname{Tr}\left(\overline{M \Sigma}_{t}\right)\right) \mathrm{d} t+2 \sqrt{\operatorname{Tr}\left(\bar{Q}^{T} \overline{\mathrm{Q}}_{t}\right)} \mathrm{d} \eta_{t},
\end{aligned}
$$

where $\xi_{t}$ and $\eta_{t}$ are the Brownian motions (Appendix).

We now determine the covariation of the stock price and Wishart processes:

$$
\begin{aligned}
\operatorname{Cov}_{t}\left(\mathrm{~d} X_{t}, \mathrm{~d} \xi_{t}\right) & =\operatorname{Cov}_{t}\left(\frac{\operatorname{Tr}\left(\sqrt{\Sigma_{t}} \mathrm{~d} Z_{t}\right)}{\sqrt{\operatorname{Tr}\left(\Sigma_{t}\right)}}, \frac{\operatorname{Tr}\left(\mathrm{Qd} W_{t} \sqrt{\Sigma_{t}}\right)}{\sqrt{\operatorname{Tr}\left(Q^{T} \mathrm{Q} \Sigma_{t}\right)}}\right) \\
& =\mathbb{E}_{t}\left(\frac{\operatorname{Tr}\left(\sqrt{\Sigma_{t}} \mathrm{~d} W_{t} R^{T}\right)}{\sqrt{\operatorname{Tr}\left(\Sigma_{t}\right)}} \frac{\operatorname{Tr}\left(\mathrm{Qd} W_{t} \sqrt{\Sigma_{t}}\right)}{\sqrt{\operatorname{Tr}\left(Q^{T} Q \Sigma_{t}\right)}}\right) \\
& =\frac{\sum_{i j} \operatorname{Cov}_{t}\left(e_{i}^{T} \sqrt{\Sigma_{t}} \mathrm{~d} W_{t} R^{T} e_{i}, e_{j}^{T} \mathrm{Qd} W_{t} \sqrt{\Sigma_{t}} e_{j}\right)}{\sqrt{\operatorname{Tr}\left(\Sigma_{t}\right)} \sqrt{\operatorname{Tr}\left(Q^{T} Q \Sigma_{t}\right)}} \\
& =\frac{\sum_{i j} \mathbb{E}_{t}\left(e_{i}^{T} \sqrt{\Sigma_{t}} \mathrm{~d} W_{t} R^{T} e_{i} e_{j}^{T} Q^{T} \mathrm{~d} W_{t}^{T} \sqrt{\Sigma_{t}} e_{j}\right)}{\sqrt{\operatorname{Tr}\left(\Sigma_{t}\right)} \sqrt{\operatorname{Tr}\left(Q^{T} Q \Sigma_{t}\right)}} \\
& =\frac{\sum_{i j} e_{i}^{T} \sqrt{\Sigma_{t}} \operatorname{Tr}\left(R^{T} e_{i} e_{j}^{T} Q^{T}\right) \sqrt{\Sigma_{t}} e_{j} \mathrm{~d} t}{\sqrt{\operatorname{Tr}\left(\Sigma_{t}\right)} \sqrt{\operatorname{Tr}\left(Q^{T} Q \Sigma_{t}\right)}} \\
& =\frac{\sum_{i j} \operatorname{Tr}\left(Q R e_{i} e_{j}^{T}\right) e_{i}^{T} \Sigma_{t} e_{j} \mathrm{~d} t}{\sqrt{\operatorname{Tr}\left(\Sigma_{t}\right)} \sqrt{\operatorname{Tr}\left(Q^{T} Q \Sigma_{t}\right)}} \\
& =\frac{\sum_{i j} e_{j}^{T} Q R e_{i} e_{i}^{T} \Sigma_{t} e_{j} \mathrm{~d} t}{\sqrt{\operatorname{Tr}\left(\Sigma_{t}\right)} \sqrt{\operatorname{Tr}\left(Q^{T} Q \Sigma_{t}\right)}} \\
& =\frac{\sum_{i j} e_{j}^{T} Q R \Sigma_{t} e_{j} \mathrm{~d} t}{\sqrt{\operatorname{Tr}\left(\Sigma_{t}\right)} \sqrt{\operatorname{Tr}\left(Q^{T} Q \Sigma_{t}\right)}} \\
\operatorname{Cov}_{t}\left(\mathrm{~d} X_{t}, \mathrm{~d} \xi_{t}\right)= & \frac{\operatorname{Tr}\left(R^{T} Q \Sigma_{t}\right)}{\sqrt{\operatorname{Tr}\left(\Sigma_{t}\right)} \sqrt{\operatorname{Tr}\left(Q^{T} Q \Sigma_{t}\right)}} . \\
& \\
&
\end{aligned}
$$

Similarly, for the second stochastic differential equation of the Wishart processes, the determination of covariation follows the same procedures, that is,

$$
\operatorname{Cov}_{t}\left(\mathrm{~d} \bar{X}_{t}, \mathrm{~d} \eta_{t}\right)=\frac{\operatorname{Tr}\left(\bar{R}^{T} \overline{\mathrm{Q}}_{t}\right)}{\sqrt{\operatorname{Tr}\left(\bar{\Sigma}_{t}\right)} \sqrt{\operatorname{Tr}\left(\overline{\mathrm{Q}}^{T} \overline{\mathrm{Q}}_{t}\right)}}
$$

Then, 
2.4. The Correlation Structure of the Model. The correlated Brownian motions $W_{t}, Z_{t}$ and $\bar{W}_{t}, \bar{Z}_{t}$, respectively, result in constant correlations $R, \bar{R} \in M_{n}$, which describe the two respective correlation structures, such that $Z_{t}$ and $\bar{Z}_{t}$ can be presented as follows:

$$
\begin{aligned}
& Z_{t}:=W_{t} R^{T}+B_{t} \sqrt{\square-R R^{T}}, \\
& \bar{Z}_{t}:=\bar{W}_{t} \bar{R}^{T}+\bar{B}_{t} \sqrt{\square-\overline{R R}^{T}},
\end{aligned}
$$

where $\square$ is the identity matrix, $T$ is the transposition, and $B_{t}$ and $\bar{B}_{t}$ are the Brownian matrices independent of $W_{t}$ and $\bar{W}_{t}$, respectively.

2.5. The Dynamic of Log-Price for the Double Wishart Model. The matrices $R$ and $\bar{R}$ describe the correlations between the Brownian of the asset price and those of the Wishart processes.

Lemma 2. The log-price dynamic $Y_{t}=\log \left(S_{t}\right)$ under the double Wishart model is given as

$$
\mathrm{d} Y_{t}=\left(r-\frac{1}{2} \operatorname{Tr}\left[\Sigma_{t}+\overline{\Sigma_{t}}\right]\right) \mathrm{d} t+\operatorname{Tr}\left[\sqrt{\Sigma_{t}} \mathrm{~d} Z_{t}+\sqrt{\overline{\Sigma_{t}}} \mathrm{~d} \bar{Z}_{t}\right]
$$

Proof. Let $Y_{t}=\log \left(S_{t}\right)$, and the asset dynamic is given as

$$
\frac{\mathrm{d} S(t)}{S(t)}=r \mathrm{~d} t+\operatorname{Tr}\left[\sqrt{\Sigma_{t}} \mathrm{~d} Z_{t}+\sqrt{\overline{\Sigma_{t}}} \mathrm{~d} \bar{Z}_{t}\right] .
$$

By applying Ito's formula on $Y_{t}$, referring the studies by Björk [18] and Shreve [19], we get

$$
\mathrm{d} Y_{t}=\operatorname{d} \log \left(S_{t}\right)=\frac{\mathrm{d} S_{t}}{S_{t}}-\frac{1}{2} \frac{\left(\mathrm{d} S_{t}\right)^{2}}{S_{t}^{2}} .
$$

Substituting the asset process (14) in the derivative expression (15) of $Y_{t}$,

$$
\begin{aligned}
\mathrm{d} Y(t) & =r \mathrm{~d} t+\operatorname{Tr}\left[\sqrt{\Sigma_{t}} \mathrm{~d} Z_{t}+\sqrt{\overline{\Sigma_{t}}} \mathrm{~d} \bar{Z}_{t}\right]-\frac{1}{2} \operatorname{Tr}\left[\Sigma_{t}+\bar{\Sigma}_{t}\right] \mathrm{d} t, \\
\mathrm{~d} Y_{t} & =\left(r-\frac{1}{2} \operatorname{Tr}\left[\Sigma_{t}+\overline{\Sigma_{t}}\right]\right) \mathrm{d} t+\operatorname{Tr}\left[\sqrt{\Sigma_{t}} \mathrm{~d} Z_{t}+\sqrt{\overline{\Sigma_{t}}} \mathrm{~d} \bar{Z}_{t}\right],
\end{aligned}
$$

which can be written as

$$
\begin{aligned}
\mathrm{d} Y(t) & =\left(r-\frac{1}{2} \operatorname{Tr}\left[\Sigma_{t}+\bar{\Sigma}_{t}\right]\right) \mathrm{d} t+\operatorname{Tr}\left[\sqrt{\Sigma_{t}}\left(\mathrm{~d} W_{t} R^{T}+\mathrm{d} B_{t} \sqrt{\square-R R^{T}}\right)+\sqrt{\bar{\Sigma}_{t}}\left(\mathrm{~d} \bar{W}_{t} \bar{R}^{T}+\mathrm{d} \bar{B}_{t} \sqrt{\square-\overline{R R}^{T}}\right)\right] \\
Y_{0} & =y .
\end{aligned}
$$

\section{Option Pricing Problem}

This part deals with the European call option pricing problem, given its payoff as

$$
\left(S_{T}-K\right)^{+} \text {. }
$$

To handle this pricing problem, the infinitesimal generator of the Wishart processes should be obtained, in order to employ the conditional characteristic function on the log asset return. The Riccati ordinary differential equations are linearized to give the closed-form solution to the pricing problem through fast Fourier transforms of Duffie et al. [15]. This is possible because the Wishart processes preserve the property of analytical tractability, since it belongs to the class of affine [20].

3.1. Infinitesimal Generator. The log-price process and Wishart processes, together with corresponding pair of two correlated Brownian motions, $Z_{s}^{Y}, Z_{s}^{\Sigma}$ and $\bar{Z}_{s}^{Y}, \bar{Z}_{s}^{\Sigma}$, can be written in the form as in the study by Benabid et al. [9], for easy handling of the complexity of the dynamics:

$$
\begin{aligned}
\mathrm{d} Y_{t} & =\left(r-\frac{1}{2} \operatorname{Tr}\left[\Sigma_{t}+\overline{\Sigma_{t}}\right]\right) \mathrm{d} t+\operatorname{Tr}\left[\sqrt{\Sigma_{t}} \mathrm{~d} Z_{t}^{Y}+\sqrt{\bar{\Sigma}_{t}} \mathrm{~d} \bar{Z}_{t}^{Y}\right], \\
\mathrm{d} \operatorname{Tr}\left(\Sigma_{t}\right) & =\left(\left(\beta \operatorname{Tr}\left(Q^{T} Q\right)\right)+2 \operatorname{Tr}\left(M \Sigma_{t}\right)\right) \mathrm{d} t+2 \sqrt{\operatorname{Tr}\left(Q^{T} Q \Sigma_{t}\right)}\left(\rho_{t} \mathrm{~d} Z_{t}^{Y}+\sqrt{\square-\rho_{t}^{2}} \mathrm{~d} Z_{t}^{\Sigma}\right), \\
\mathrm{d} \operatorname{Tr}\left(\bar{\Sigma}_{t}\right) & =\left(\left(\bar{\beta} \operatorname{Tr}\left(\overline{\mathrm{Q}}^{T} \overline{\mathrm{Q}}\right)\right)+2 \operatorname{Tr}\left(\overline{M \Sigma_{t}}\right)\right) \mathrm{d} t+2 \sqrt{\operatorname{Tr}\left(\overline{\mathrm{Q}}^{T} \overline{\mathrm{Q}} \Sigma_{t}\right)}\left(\bar{\rho}_{t} \mathrm{~d} \bar{Z}_{t}^{Y}+\sqrt{\square-\bar{\rho}_{t}^{2}} \mathrm{~d} \bar{Z}_{t}^{\bar{\Sigma}}\right),
\end{aligned}
$$


where

$$
\begin{aligned}
\mathrm{d}\left\langle Z^{Y}, Z^{\Sigma}\right\rangle_{t} & =\rho_{t}=\frac{\operatorname{Tr}\left(R^{T} \mathrm{Q} \Sigma_{t}\right)}{\sqrt{\operatorname{Tr}\left(\Sigma_{t}\right)} \sqrt{\operatorname{Tr}\left(\mathrm{Q}^{T} \mathrm{Q} \Sigma_{t}\right)}} \\
\mathrm{d}\left\langle\bar{Z}^{Y}, \bar{Z}^{\bar{\Sigma}}\right\rangle_{t} & =\bar{\rho}_{t}=\frac{\operatorname{Tr}\left(\bar{R}^{T} \overline{\left.\mathrm{Q} \Sigma_{t}\right)}\right.}{\sqrt{\operatorname{Tr}\left(\bar{\Sigma}_{t}\right)} \sqrt{\operatorname{Tr}\left(\overline{\mathrm{Q}}^{T} \overline{\mathrm{Q} \Sigma_{t}}\right)}} .
\end{aligned}
$$

Proposition 1. The infinitesimal generator of the double Wishart stochastic volatility model for $\left(Y_{t}, \Sigma_{t}, \bar{\Sigma}_{t}\right)$ is given by

$$
\begin{aligned}
\mathscr{L}_{Y, \Sigma, \bar{\Sigma}}= & \left(r-\frac{\operatorname{Tr}[\Sigma+\bar{\Sigma}]}{2}\right) \frac{\partial}{\partial y}+\frac{\operatorname{Tr}[\Sigma+\bar{\Sigma}]}{2} \frac{\partial^{2}}{\partial y^{2}} \\
& +\left(\beta \operatorname{Tr}\left(Q^{T} Q\right)+2 \operatorname{Tr}(M \Sigma)\right) \frac{\partial}{\partial \Sigma}+2 \operatorname{Tr}\left(\Sigma \frac{\partial}{\partial \Sigma} Q^{T} Q \frac{\partial}{\partial \Sigma}\right) \\
& +\left(\bar{\beta} \operatorname{Tr}\left(\bar{Q}^{T} \bar{Q}\right)+2 \operatorname{Tr}(\overline{M \Sigma})\right) \frac{\partial}{\partial \bar{\Sigma}}+2 \operatorname{Tr}\left(\bar{\Sigma} \frac{\partial}{\partial \bar{\Sigma}} \bar{Q}^{T} \bar{Q} \frac{\partial}{\partial \bar{\Sigma}}\right) \\
& +2 \operatorname{Tr}\left(\Sigma R Q \frac{\partial}{\partial \Sigma}\right) \frac{\partial}{\partial y}+2 \operatorname{Tr}\left(\bar{\Sigma} R \bar{Q} \frac{\partial}{\partial \bar{\Sigma}}\right) \frac{\partial}{\partial y} .
\end{aligned}
$$

Proof. The infinitesimal generator has a nontrivial term which arises from covariation $\mathrm{d}\left\langle\Sigma_{\theta}^{i j}, Y\right\rangle$ corresponding to the coefficients of the term

$$
\frac{\partial^{2}}{\partial x_{\theta ; i j} \partial y}=\frac{\partial}{\partial x_{\theta ; i j}}\left(\frac{\partial}{\partial y}\right), \quad i, j=1, \ldots, n, \theta=1,2 .
$$

Let $V_{\theta ; t}:=\sqrt{\sum_{\theta ; t}}$ be the square root matrix, such that

$$
\Sigma_{\theta ; t}^{i j}=\sum_{t=1}^{n} V_{\theta ; t}^{i l} V_{\theta ; t}^{l j}=\sum_{t=1}^{n} V_{\theta ; t}^{i l} V_{\theta ; t}^{j l} .
$$

Due to the fact that $V_{\theta ; t}$ is symmetric, we find the covariation terms matching with $\partial^{2} / \partial x_{\theta ; i j} \partial y$ coefficients:

$$
\begin{aligned}
\left\langle\mathrm{d} \Sigma_{\theta}^{i j}, Y\right\rangle & =\mathbb{E}_{t}\left[\left(\sum_{i, k=1}^{n} V_{\theta ; t}^{i l} \mathrm{~d} W_{l k}^{\theta} Q_{k j}^{\theta}+\sum_{l, k=1}^{n} V_{\theta ; t}^{j l} \mathrm{~d} W_{l k}^{\theta} Q_{k i}^{\theta}\right)\left(\sum_{l, k, h=1}^{n} V_{\theta ; t}^{l k} \mathrm{~d} W_{k h}^{\theta} R_{l h}^{\theta}\right)\right] \\
& =\sum_{l, k, h=1}^{n}\left(V_{\theta ; t}^{i l} Q_{k j}^{\theta}+V_{\theta ; t}^{j l} Q_{k i}^{\theta}\right) V_{\theta ; t}^{h l} R_{h k}^{\theta} \mathrm{d} t \\
& =\sum_{k, h=1}^{n}\left[\left(\sum_{l=1}^{n} V_{\theta ; t}^{i l} V_{\theta ; t}^{h l}\right) Q_{k j}^{\theta}+\left(\sum_{l=1}^{n} V_{\theta ; t}^{j l} V_{\theta ; t}^{h l}\right) Q_{k i}^{\theta}\right] R_{h k}^{\theta} \mathrm{d} t \\
& =\sum_{k, h=1}^{n}\left(\sum_{\theta ; t}^{i h} Q_{k j}^{\theta}+\Sigma_{\theta ; t}^{j h} Q_{k i}^{\theta}\right) R_{h k}^{\theta} \mathrm{d} t .
\end{aligned}
$$

This gives the corresponding coefficients of the term since

$$
2 \operatorname{Tr}\left(\Sigma_{\theta} R^{\theta} Q^{\theta} D_{\theta}\right) \frac{\partial}{\partial y}=2 \sum_{i, j, k, h=1}^{n} D_{\theta}^{i j} \Sigma_{\theta}^{j h} R_{h k}^{\theta} Q_{k i}^{\theta} \frac{\partial}{\partial y} .
$$

The notation when $\theta=1, \Sigma_{1}=\Sigma, R^{1}=R, Q^{1}=Q, D_{1}=$ $\left(\partial / \partial x_{i j}\right)$ for $\quad \theta=2, \Sigma_{2}=\bar{\Sigma}, R^{2}=\bar{R}, Q^{2}=\bar{Q}, D_{2}=$ $\left(\partial / \partial \bar{x}_{i j}\right)$ 
3.2. The Laplace Transform of Asset Returns. Following Duffie et al. [15] and Da Fonseca et al. [3], in order to solve the European options pricing problem, we consider the Laplace transform of the process (17). The Laplace transform of the Wishart process is exponentially affine as in the study by Bru [11]; hence, the conditional moment generating function of the log-asset returns is the exponential of an affine combination of $Y$ and Wishart components, so we provide for the deterministic functions $\lambda_{1}(t), \lambda_{2}(t) \in M_{n}$, and $\delta(t), \varepsilon(t) \in \mathbb{R}$, as parameters of the Laplace transform:

$$
\begin{aligned}
\psi_{\gamma, t}(\tau) & =\mathbb{E}\left(e^{\gamma Y_{t+\tau}}\right) \\
& =\exp \left\{\operatorname{Tr} \lambda_{1}(\tau) \Sigma_{t}+\operatorname{Tr} \lambda_{2}(\tau) \bar{\Sigma}_{t}+\delta(\tau) Y_{t}+\varepsilon(\tau)\right\},
\end{aligned}
$$

where $\gamma \in \mathbb{R}$ and $\mathbb{E}_{t}$ denote the conditional expected value with respect to the probability measure.
Using Feynman-Kac argument helps us to get matrix Riccati equations:

$$
\left\{\begin{array}{l}
\frac{\partial \psi_{\gamma, t}}{\partial \tau}=\mathscr{L}_{Y, \Sigma, \Sigma} \psi_{\gamma, t} \\
\psi_{\gamma, t}(0)=\exp \left\{\gamma Y_{t}\right\} .
\end{array}\right.
$$

Proposition 2. The Laplace transform of the asset returns

$$
\psi_{\gamma, t}(\tau)=\exp \left\{\operatorname{Tr}\left[\lambda_{1}(\tau) \Sigma_{t}+\lambda_{2}(\tau) \bar{\Sigma}_{t}\right]+\delta(\tau) Y_{t}+\varepsilon(\tau)\right\},
$$

where $\lambda_{1}, \lambda_{2}$, and $\varepsilon$ are the solutions of the differential equations through linearization methods:

$$
\begin{aligned}
& \lambda_{1}^{\prime}(\tau)=M \lambda_{1}(\tau)+\left(M^{T}+2 \gamma R Q\right) \lambda_{1}(\tau)+2 \lambda_{1}(\tau) Q^{T} Q \lambda_{1}(\tau)+\frac{\gamma(\gamma-1)}{2} \mathbb{q}_{n}, \\
& \lambda_{2}^{\prime}(\tau)=\bar{M} \lambda_{2}(\tau)+\left(\bar{M}^{T}+2 \gamma \overline{R Q}\right) \lambda_{2}(\tau)+2 \lambda_{2}(\tau) \bar{Q}^{T} \bar{Q} \lambda_{2}(\tau)+\frac{\gamma(\gamma-1)}{2} \mathbb{V}_{n}, \\
& \varepsilon^{\prime}(\tau)=r \gamma+\beta \operatorname{Tr}\left[\left(Q^{T} Q\right) \lambda_{1}(\tau)\right]+\bar{\beta} \operatorname{Tr}\left[\left(\bar{Q}^{T} \bar{Q}\right) \lambda_{2}(\tau)\right],
\end{aligned}
$$

with boundary conditions $\lambda_{1}(0)=0, \lambda_{2}(0)=0, \varepsilon(0)=$ 0 , and $\delta_{(\tau)}=\gamma=C_{0}$.

$$
\begin{aligned}
& \lambda_{1}(\tau)=H_{1}(\tau)^{-1} H_{2}(\tau), \\
& \lambda_{2}(\tau)=I_{1}^{-1}(\tau) I_{2}(\tau), \\
& \mathcal{E}(\tau)=-\frac{\beta}{2} \operatorname{Tr}\left[\log H_{1}(\tau)+\left(M^{T}+2 \gamma R Q\right) \tau\right]-\frac{\bar{\beta}}{2} \operatorname{Tr}\left[\log I_{1}(\tau)+\left(\bar{M}^{T}+2 \gamma \overline{R Q}\right) \tau\right]+r \gamma \tau,
\end{aligned}
$$

with

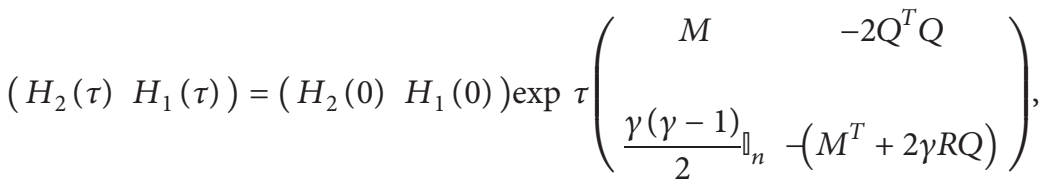

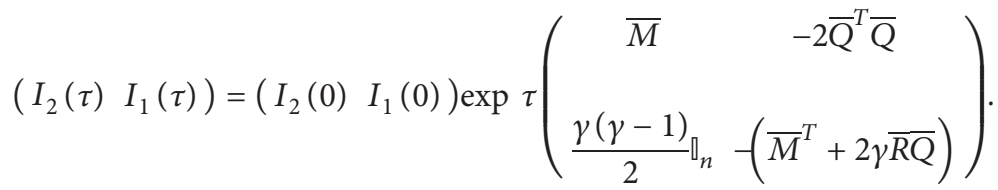


Proof

From equation (27) and the Laplace transform (26), we solve the problem by considering Proposition 1 for the double Wishart stochastic volatility model as follows:

$$
\begin{aligned}
\frac{\partial \psi_{\gamma, t}}{\partial \tau}= & \left(r-\frac{\operatorname{Tr}[\Sigma+\bar{\Sigma}]}{2}\right) \frac{\partial \psi_{\gamma, t}}{\partial y}+\frac{\operatorname{Tr}[\Sigma+\bar{\Sigma}]}{2} \frac{\partial^{2} \psi_{\gamma, t}}{\partial y^{2}} \\
& +\left(\beta \operatorname{Tr}\left(Q^{T} Q\right)+2 \operatorname{Tr}(M \Sigma)\right) \frac{\partial \psi_{\gamma, t}}{\partial \Sigma}+2 \operatorname{Tr}\left(\Sigma \frac{\partial}{\partial \Sigma} Q^{T} Q \frac{\partial}{\partial \Sigma}\right) \psi_{\gamma, t} \\
& +\left(\bar{\beta} \operatorname{Tr}\left(\bar{Q}^{T} \bar{Q}\right)+2 \operatorname{Tr}(\overline{M \Sigma})\right) \frac{\partial \psi_{\gamma, t}}{\partial \bar{\Sigma}}+2 \operatorname{Tr}\left(\bar{\Sigma} \frac{\partial}{\partial \bar{\Sigma}} \bar{Q}^{T} \bar{Q} \frac{\partial}{\partial \bar{\Sigma}}\right) \psi_{\gamma, t} \\
& +2 \operatorname{Tr}\left(\Sigma R Q \frac{\partial}{\partial \Sigma}\right) \frac{\partial \psi_{\gamma, t}}{\partial y}+2 \operatorname{Tr}\left(\overline{\Sigma R} \bar{Q} \frac{\partial}{\partial \bar{\Sigma}}\right) \frac{\partial \psi_{\gamma, t}}{\partial y} .
\end{aligned}
$$

From equation (27), we have

$$
\begin{gathered}
\lambda_{1}(0)=0, \\
\lambda_{2}(0)=0, \\
\delta(0)=\gamma, \\
\varepsilon(0)=0 .
\end{gathered}
$$

Then,

$$
\begin{aligned}
& \frac{\partial \psi_{\gamma, t}(\tau)}{\partial \tau}=\operatorname{Tr}\left\{\left(\frac{\mathrm{d} \lambda_{1}(\tau)}{\mathrm{d} \tau} \Sigma_{t}+\frac{\mathrm{d} \lambda_{2}(\tau)}{\partial \tau} \bar{\Sigma}_{t}\right)+\frac{\mathrm{d}}{\mathrm{d} \tau} \delta(t) Y_{t}+\frac{\mathrm{d}}{\mathrm{d} \tau} \varepsilon(\tau)\right\} \psi_{\gamma, t}(\tau) \\
& =\left(r-\frac{[\Sigma+\bar{\Sigma}]}{2}\right) \delta(\tau) \psi_{\gamma, t}+\frac{\operatorname{Tr}(\Sigma+\bar{\Sigma})}{2} \delta^{2}(\tau) \psi_{\gamma, t} \\
& +\left[\left(\beta \operatorname{Tr}\left(Q^{T} Q\right)+2 \operatorname{Tr}(M \Sigma)\right) \lambda_{1}(\tau)+\left(\bar{\beta} \operatorname{Tr}\left(\bar{Q}^{T} \bar{Q}\right)+2 \operatorname{Tr}(\overline{M \Sigma})\right) \lambda_{2}(\tau)\right] \psi_{\gamma, t} \\
& +2 \operatorname{Tr}\left[\Sigma \lambda_{1}(\tau) Q^{T} Q \lambda_{1}(\tau)\right] \psi_{\gamma, t}(\tau)+2 \operatorname{Tr}\left[\bar{\Sigma} \lambda_{1}(\tau) \bar{Q}^{T} \bar{Q} \lambda_{1}(\tau)\right] \psi_{\gamma, t} \\
& +2 \operatorname{Tr}\left[\Sigma R Q \delta(\tau) \lambda_{1}(\tau)\right] \psi_{\gamma, t}+2 \operatorname{Tr}\left[\bar{\Sigma} \overline{R Q} \delta(\tau) \lambda_{1}(\tau)\right] \psi_{\gamma, t} \\
& -\left[\operatorname{Tr}\left(\lambda_{1}(\tau) \Sigma_{t}+\lambda_{2}(\tau) \bar{\Sigma}_{t}\right)+\dot{\delta}(\tau) Y_{t}+\dot{\varepsilon}(\tau)\right]+\left(r-\frac{\operatorname{Tr}[\Sigma+\bar{\Sigma}]}{2}\right) \delta(\tau) \\
& +\frac{\operatorname{Tr}[\Sigma+\bar{\Sigma}]}{2} \delta^{2}(\tau)+\operatorname{Tr}\left[\left(\beta Q^{T} Q+M \Sigma+\Sigma M^{T}\right) \lambda_{1}(\tau)+\left(\bar{\beta}^{T} \bar{Q}+\overline{M \Sigma}+\overline{\Sigma M^{T}}\right) \lambda_{2}(\tau)+2 \Sigma \lambda_{1}(\tau) Q^{T} Q \lambda_{1}(\tau)\right. \\
& +2 \bar{\Sigma} \lambda_{2}\left((\tau) \bar{Q}^{T} \bar{Q} \lambda_{2}(\tau)+2 \Sigma R Q \delta(\tau) \lambda_{1}(\tau)+2 \bar{\Sigma} R \bar{Q} \delta(\tau) \lambda_{2}(\tau)\right]=0, \\
& -\operatorname{Tr}\left(\frac{\mathrm{d}}{\mathrm{d} \tau} \lambda_{1}(\tau) \Sigma\right)-\frac{1}{2} \operatorname{Tr}[\Sigma] \delta(\tau)+\frac{1}{2} \operatorname{Tr}[\Sigma] \delta^{2}(\tau) \\
& +\operatorname{Tr}\left[\left(M \Sigma+\Sigma M^{T}\right) \lambda_{1}(\tau)+2 \Sigma \lambda_{1}(\tau) Q^{T} Q \lambda_{1}(\tau)+2 \Sigma R Q \delta(\tau) \lambda_{1}(\tau)\right]=0, \\
& -\operatorname{Tr}\left(\frac{\mathrm{d}}{\mathrm{d} \tau} \lambda_{2}(\tau) \bar{\Sigma}\right)-\frac{1}{2} \operatorname{Tr}[\bar{\Sigma}] \delta(\tau)+\frac{1}{2} \operatorname{Tr}[\bar{\Sigma}] \delta^{2}(\tau) \\
& +\operatorname{Tr}\left[\left(\bar{M} \Sigma+\overline{\Sigma M}^{T}\right) \lambda_{2}(\tau)+2 \bar{\Sigma} \lambda_{2}(\tau) \bar{Q}^{T} \bar{Q} \lambda_{2}(\tau)+2 \overline{\Sigma \bar{R} Q} \delta(\tau) \lambda_{2}(\tau)\right]=0 \\
& -\delta(\tau) Y_{t}-\dot{\varepsilon}(\tau)+\mu \delta(\tau)+\operatorname{Tr}\left[\beta Q^{T} Q\right] \lambda_{1}(\tau)+\operatorname{Tr}\left[\bar{\beta}^{T} \bar{Q}\right] \lambda_{2}(\tau)=0
\end{aligned}
$$


Now, we need to identify the coefficients for above equations and obtain the matrix Riccati ordinary differential equations:

$$
\begin{aligned}
& \left\{\begin{array}{l}
\frac{\mathrm{d}}{\mathrm{d} \tau} \lambda_{1}(\tau)=M \lambda_{1}(\tau)+\left(M^{T}+2 \gamma R Q\right) \lambda_{1}(\tau)+2 \lambda_{1}(\tau) Q^{T} Q \lambda_{1}(\tau)+\frac{\gamma(\gamma-1)}{2} \mathbb{Q}_{n} \\
\lambda_{1}(0)=0
\end{array}\right. \\
& \left\{\begin{array}{l}
\frac{\mathrm{d}}{\mathrm{d} \tau} \lambda_{2}(\tau)=\bar{M} \lambda_{2}(\tau)+\left(\bar{M}^{T}+2 \gamma \bar{R} Q\right) \lambda_{2}(\tau)+2 \lambda_{2}(\tau) \bar{Q}^{T} \bar{Q} \lambda_{2}(\tau)+\frac{\gamma(\gamma-1)}{2} \mathbb{a}_{n} \\
\lambda_{2}(0)=0
\end{array}\right.
\end{aligned}
$$

For the constant $\varepsilon$, the matrix Riccati ordinary differential is obtained as follows:

$$
\begin{aligned}
& -\delta^{\prime}(\tau) Y_{t}-\varepsilon^{\prime}(\tau)+r \delta(\tau)+\operatorname{Tr}\left(\beta Q^{T} Q\right) \lambda_{1}(\tau)+\operatorname{Tr}\left(\bar{\beta} \bar{Q}^{T} \bar{Q}\right) \lambda_{2}(\tau)=0, \\
& \delta(\tau)=C_{0}=\gamma, \text { since } \delta^{\prime}(\tau)=0 \text { so } \Rightarrow \delta(\tau)=\gamma \\
& \left\{\begin{array}{l}
\frac{\mathrm{d} \varepsilon(\tau)}{\mathrm{d} \tau}=r \gamma+\beta \operatorname{Tr}\left[\left(Q^{T} Q\right) \lambda_{1}(\tau)\right]+\bar{\beta} \operatorname{Tr}\left[\left(\bar{Q}^{T} \bar{Q}\right) \lambda_{2}(\tau)\right] \\
\varepsilon(0)=0 .
\end{array}\right.
\end{aligned}
$$

Then, $\varepsilon(\tau)$ is obtained by integrating directly

$$
\varepsilon(\tau)=\int_{0}^{\tau} r \gamma \mathrm{d} s+\int_{0}^{\tau} \operatorname{Tr}\left[\beta Q^{T} Q \lambda_{1}(s)+\bar{\beta} \bar{Q}^{T} \bar{Q} \lambda_{2}(s)\right] \mathrm{d} s,
$$

with $\lambda_{1}, \lambda_{2} \in M_{n}(\mathbb{R})$, and $\delta(\tau), \varepsilon(\tau) \in \mathbb{R}$.

The matrix Riccati equations above are linearized to provide the closed-form solution, following the techniques of Benabid et al. [9] and Da Fonseca et al. [3]. So from equations (35) and (36), let

$$
\lambda_{1}(\tau)=H_{1}(\tau)^{-1} H_{2}(\tau)
$$

where $H_{1}(\tau) \in G L_{n}(\mathbb{R}), H_{2}(\tau) \in M_{n}(\mathbb{R})$, and therefore,

$$
\begin{aligned}
& \frac{\mathrm{d}}{\mathrm{d} \tau}\left[H_{1}(\tau) \lambda_{1}(\tau)\right]=\frac{\mathrm{d} H_{1}(\tau)}{\mathrm{d} \tau} \lambda_{1}(\tau)+H_{1}(\tau) \frac{\mathrm{d} \lambda_{1}(\tau)}{\mathrm{d} \tau}, \\
& H_{1}(\tau) \frac{\mathrm{d} \lambda_{1}(\tau)}{\mathrm{d} \tau}=\frac{\mathrm{d}}{\mathrm{d} \tau}\left[H_{1}(\tau) \lambda_{1}(\tau)\right]-\frac{\mathrm{d} H_{1}(\tau)}{\mathrm{d} \tau} \lambda_{1}(\tau), \\
& H_{1}(\tau) \lambda_{1}(\tau) M+H_{1}(\tau)\left(M^{T}+2 \gamma R Q\right) \lambda_{1}(\tau)+2 H_{1}(\tau) \lambda_{1}(\tau) Q^{T} Q \lambda_{1}(\tau) \\
& \quad+H_{1}(\tau) \frac{\gamma(\gamma-1)}{2}=H_{1}(\tau) \frac{\mathrm{d} \lambda_{1}(\tau)}{\mathrm{d} \tau}, \\
& H_{2}(\tau) M+H_{1}(\tau)\left(M^{T}+2 \gamma R Q\right) \lambda_{1}(\tau)+2 H_{2}(\tau) Q^{T} Q \lambda_{2}(\tau) \\
& \quad+H_{1}(\tau) \frac{\gamma(\gamma-1)}{2}=H_{1}(\tau) \frac{\mathrm{d} \lambda_{1}(\tau)}{\mathrm{d} \tau} .
\end{aligned}
$$


Since $H_{2}(\tau)=H_{1}(\tau) \lambda_{1}(\tau)$, now we have

$$
\frac{\mathrm{d} H_{2}(\tau)}{\mathrm{d} \tau}=\frac{\mathrm{d} H_{1}(\tau)}{\mathrm{d} \tau} \lambda_{1}(\tau)+H_{1}(\tau) \frac{\mathrm{d} \lambda_{1}(\tau)}{\mathrm{d} \tau} .
$$

$$
H_{1}(\tau) \frac{\mathrm{d} \lambda_{1}(\tau)}{\mathrm{d} \tau}=\frac{\mathrm{d} H_{2}(\tau)}{\mathrm{d} \tau}-\frac{\mathrm{d} H_{1}(\tau)}{\mathrm{d} \tau} \lambda_{1}(\tau)
$$

Then, we get expressions

This implies that

$$
\begin{aligned}
\frac{\mathrm{d} H_{2}(\tau)}{\mathrm{d} \tau}-\frac{\mathrm{d} H_{1}(\tau)}{\mathrm{d} \tau} \lambda_{1}(\tau) & =H_{2}(\tau) M+H_{1}(\tau)\left(M^{T}+2 \gamma R Q\right) \lambda_{1}(\tau)+2 H_{2}(\tau) Q^{T} Q \lambda_{1}(\tau)+H_{1}(\tau) \frac{\gamma(\gamma-1)}{2} \\
& =H_{1}(\tau) \frac{\gamma(\gamma-1)}{2}+H_{2}(\tau) M+\left[H_{1}(\tau)\left(M^{T}+2 \gamma R Q\right)+2 H_{2}(\tau) Q^{T} Q\right] \lambda_{1}(\tau), \\
\frac{\mathrm{d} H_{2}(\tau)}{\mathrm{d} \tau} & =H_{1}(\tau) \frac{\gamma(\gamma-1)}{2}+H_{2}(\tau) M, \\
\frac{\mathrm{d} H_{1}(\tau)}{\mathrm{d} \tau} & =-H_{1}(\tau)\left(M^{T}+2 \gamma R Q\right)-2 H_{2}(\tau) Q^{T} Q \\
\frac{\mathrm{d}}{\mathrm{d} \tau}\left(H_{2}(\tau) H_{1}(\tau)\right) & =\left(H_{2}(\tau) H_{1}(\tau)\right)\left(\begin{array}{cc}
M & -2 Q^{T} Q \\
\frac{\gamma(\gamma-1)}{2} \mathbb{v}_{n}-\left(M^{T}+2 \gamma R Q\right)
\end{array}\right) .
\end{aligned}
$$

The solution of differential equation (46) above is obtained through exponentiation as follows:

$$
\left(H_{2}(\tau) H_{1}(\tau)\right)=\left(H_{2}(0) H_{1}(0)\right) e^{Q \tau},
$$

with conditions $H_{1}(0)=K \rrbracket_{n}$ and $H_{1}^{-1}(0)=K^{-1} \rrbracket_{n}$ and for $K=1, H_{1}^{-1}(0)=\mathbb{q}_{n}$, such that

$$
\left(H_{2}(\tau) H_{1}(\tau)\right)=\left(\lambda_{1}(0) \mathbb{q}_{n}\right) e^{\tau Q},
$$

where

$$
e^{\tau Q}=\left(\begin{array}{cc}
\lambda_{1}^{11}(\tau) & \lambda_{1}^{12}(\tau) \\
\lambda_{1}^{21}(\tau) & \lambda_{1}^{22}(\tau)
\end{array}\right)
$$

Then,

$$
\begin{aligned}
\left(\begin{array}{lll}
H_{2}(\tau) & H_{1}(\tau)
\end{array}\right) & =\left(\begin{array}{lll}
\lambda_{1}(0) & \mathbb{q}_{n}
\end{array}\right)\left(\begin{array}{cc}
\lambda_{1}^{11}(\tau) & \lambda_{1}^{12}(\tau) \\
\lambda_{1}^{21}(\tau) & \lambda_{1}^{22}(\tau)
\end{array}\right) \\
& =\left[\begin{array}{lll}
\lambda_{1}(0) & \lambda_{1}^{11}(\tau)+\lambda_{1}^{21}(\tau) & \lambda_{1}(0) \lambda_{1}^{12}(\tau)+\lambda_{1}^{22}(\tau)
\end{array}\right]
\end{aligned}
$$

and since $\lambda_{1}(0)=0$, then

$$
\left(H_{2}(\tau) H_{1}(\tau)\right)=\left(\lambda_{1}^{21}(\tau) \lambda_{1}^{22}(\tau)\right) .
$$

In conclusion, we get

$$
\lambda_{1}(\tau)=\lambda_{1}^{22}(\tau)^{-1} \lambda_{1}^{21}(\tau)
$$

This represents the closed-form solution of the Riccati equation (35).

Now, we look at the solution of the second Riccati equation (36). Let

$$
\lambda_{2}(\tau)=I_{1}^{-1}(\tau) I_{2}(\tau)
$$

then,

$$
\begin{aligned}
& \frac{\mathrm{d} I_{2}(\tau)}{\mathrm{d} \tau}=I_{1}(\tau) \frac{\gamma(\gamma-1)}{2}+I_{2}(\tau) \bar{M}, \\
& \frac{\mathrm{d} I_{1}(\tau)}{\mathrm{d} \tau}=-I_{1}(\tau)\left(\bar{M}^{T}+2 \gamma \bar{R} \bar{Q}\right)-2 I_{2}(\tau) \bar{Q}^{T} \bar{Q},
\end{aligned}
$$




$$
\frac{\mathrm{d}}{\mathrm{d} \tau}\left(I_{2}(\tau) I_{1}(\tau)\right)=\left(\begin{array}{ll}
I_{2}(\tau) & \left.I_{1}(\tau)\right)
\end{array}\right)\left(\begin{array}{cc}
\bar{M} & -2 \overline{\mathrm{Q}}^{T} \overline{\mathrm{Q}} \\
\frac{\gamma(\gamma-1)}{2} \rrbracket_{n} & -\left(\bar{M}^{T}+2 \gamma \overline{R Q}\right)
\end{array}\right),
$$

such that

$$
\left(I_{2}(\tau) I_{1}(\tau)\right)=\left(I_{2}(0) I_{1}(0)\right) e^{\overline{\mathrm{Q}} \tau}
$$

From equation (40), we go through the same procedures as above, with conditions

$$
\begin{aligned}
& I_{2}(0)=I_{1}(0) \lambda_{2}(0)=\lambda_{2}(0), \\
& I_{1}(0)=\bar{K} \rrbracket=\bar{K}=\rrbracket
\end{aligned}
$$

therefore,

$$
\begin{aligned}
& \left(\begin{array}{lll}
I_{2}(\tau) & I_{1}(\tau)
\end{array}\right)=\left(\begin{array}{lll}
\lambda_{2}(0) & \mathbb{q}_{n}
\end{array}\right)\left(\begin{array}{ll}
\lambda_{2}^{11}(\tau) & \lambda_{2}^{12}(\tau) \\
\lambda_{2}^{21}(\tau) & \lambda_{2}^{22}(\tau)
\end{array}\right), \\
& \left(I_{2}(\tau) I_{1}(\tau)\right)=\left(\lambda_{2}(0) \lambda_{2}^{11}(\tau)+\lambda_{2}^{21}(\tau) \lambda_{2}(0) \lambda_{2}^{12}(\tau)+\lambda_{2}^{22}(\tau)\right),
\end{aligned}
$$

since $\lambda_{2}(0)=0$, then

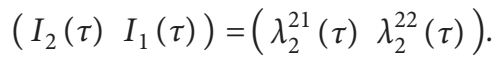

$$
\left\{\begin{array}{l}
\frac{\mathrm{d} \varepsilon(\tau)}{\mathrm{d} \tau}=r \gamma+\beta \operatorname{Tr}\left[\left(Q^{T} Q\right) \lambda_{1}(\tau)\right]+\bar{\beta} \operatorname{Tr}\left[\left(\bar{Q}^{T} \bar{Q}\right) \lambda_{2}(\tau)\right] \\
\varepsilon(0)=0
\end{array}\right.
$$

Thus, it provides the expression for a closed-form solution of the Riccati equation:

$$
\lambda_{2}(\tau)=\lambda_{2}^{22}(\tau)^{-1} \lambda_{2}^{21}(\tau)
$$

From Riccati equations (35) and (36), we have

Let compute the last Riccati equation (37) for constant $\varepsilon$,

$$
\begin{gathered}
\left\{\begin{array}{l}
\frac{\mathrm{d} H_{2}(\tau)}{\mathrm{d} \tau}=H_{1}(\tau) \frac{\gamma(\gamma-1)}{2}+H_{2}(\tau) M, \\
\frac{\mathrm{d} H_{1}(\tau)}{\mathrm{d} \tau}=-H_{1}(\tau)\left(M^{T}+2 \gamma R Q\right)-2 H_{2}(\tau) Q^{T} Q,
\end{array}\right. \\
\left\{\begin{array}{l}
\frac{\mathrm{d} I_{2}(\tau)}{\mathrm{d} \tau}=I_{1}(\tau) \frac{\gamma(\gamma-1)}{2}+I_{2}(\tau) \bar{M}, \\
\frac{\mathrm{d} I_{1}(\tau)}{\mathrm{d} \tau}=-I_{1}(\tau)\left(\bar{M}^{T}+2 \gamma \overline{R Q}\right)-2 I_{2}(\tau) \bar{Q}^{T} \bar{Q},
\end{array}\right. \\
\frac{\mathrm{d} \varepsilon(\tau)}{\mathrm{d} \tau}=r \gamma+\beta \operatorname{Tr}\left[\left(Q^{T} Q\right) H_{1}^{-1}(\tau) H_{2}(\tau)\right]+\bar{\beta} \operatorname{Tr}\left[\left(\bar{Q}^{T} \bar{Q}\right) I_{1}^{-1}(\tau) I_{2}(\tau)\right] .
\end{gathered}
$$

From equation (63), we get

$$
H_{2}(\tau)=-\frac{1}{2}\left[\frac{\mathrm{d} H_{1}(\tau)}{\mathrm{d} \tau}+H_{1}(\tau)\left(M^{T}+2 \gamma R Q\right)\right]\left(Q^{T} Q\right)^{-1}
$$

and as well as from equation (64),

$$
I_{2}(\tau)=-\frac{1}{2}\left[\frac{\mathrm{d} I_{1}(\tau)}{\mathrm{d} \tau}+I_{1}(\tau)\left(\bar{M}^{T}+2 \gamma \bar{R} \bar{Q}\right)\right]\left(\bar{Q}^{T} \bar{Q}\right)^{-1},
$$


and then, substituting equations (66) and (67) in (65) provides

$$
\begin{gathered}
\left\{\frac{\mathrm{d} \varepsilon(\tau)}{\mathrm{d} \tau}=-\frac{\beta}{2} \operatorname{Tr}\left[H_{1}^{-1}(\tau) \frac{\mathrm{d} H_{1}(\tau)}{\mathrm{d} \tau}+\left(M^{T}+2 \gamma R Q\right)\right]-\frac{\bar{\beta}}{2} \operatorname{Tr}\left[I_{1}^{-1}(\tau) \frac{\mathrm{d} I_{1}(\tau)}{\mathrm{d} \tau}+\left(\bar{M}^{T}+2 \gamma \overline{R Q}\right)\right]+r \gamma, \varepsilon(0)=0\right. \\
\mathrm{d} \varepsilon(\tau)=-\frac{\beta}{2} \operatorname{Tr}\left[\frac{\mathrm{d} H_{1}(\tau)}{H_{1}(\tau)}+\left(M^{T}+2 \gamma R Q\right) \mathrm{d} \tau\right]-\frac{\bar{\beta}}{2} \operatorname{Tr}\left[\frac{\mathrm{d} I_{1}(\tau)}{I_{1}(\tau)}+\left(\bar{M}^{T}+2 \gamma \overline{R Q}\right) \mathrm{d} \tau\right]+r \gamma \mathrm{d} \tau .
\end{gathered}
$$

By integrating (69), we obtain

$$
\varepsilon(\tau)=-\frac{\beta}{2} \operatorname{Tr}\left[\log H_{1}(\tau)+\left(M^{T}+2 \gamma R Q\right) \tau\right]-\frac{\bar{\beta}}{2} \operatorname{Tr}\left[\log I_{1}(\tau)+\left(\bar{M}^{T}+2 \gamma \overline{R Q}\right) \tau\right]+r \gamma \tau
$$

3.3. Fast Fourier Transform Method and Characteristic Function. In this section, we consider the fast Fourier transform (FFT) method in the study by Carr and Madan [16] to price European call option with $\alpha>0$, at time $t$, strike $k=\log (K)$, and time to maturity $T$, given as

$$
\begin{aligned}
& C_{t}(T, K)=e^{-r(T-t)} \mathbb{E}\left[\left(X_{T}-K\right)^{+} \mid \mathscr{F}_{t}\right], \\
& C_{t}(T, K)=C_{t}(T, k)=e^{-r(T-t)} \mathbb{E}\left[\left(\exp \left(Y_{t}\right)-\exp (k)\right)^{+} \mid \mathscr{F}_{t}\right] .
\end{aligned}
$$

The modified price $C_{t}^{\alpha}$ in the study by Carr and Madan [16] is considered, with $\alpha=1.1$ as a good empirical value for the Heston model. The square integrable function is obtained through the modified price in order to use inverse Fourier transform:

$$
C_{t}^{\alpha}(T, k)=\exp (\alpha k) C_{t}(T, K) .
$$

We introduce the Fourier transform of the modified price together with the application of the Fubini integration theorem:

$$
\begin{aligned}
\psi_{t}^{\alpha}(T, \theta) & =\int_{-\infty}^{+\infty} C_{t}^{\alpha}(T, k) e^{i \theta k} \mathrm{~d} k \\
& =\int_{-\infty}^{+\infty} e^{\alpha k} C_{t}(T, K) e^{i \theta k} \mathrm{~d} k \\
& =\int_{-\infty}^{+\infty} e^{\alpha k} e^{-r(T-t)} \mathbb{E}\left[\left(\exp \left(Y_{t}\right)-\exp (k)\right)^{+} \mid \mathscr{F}_{t}\right] e^{i \theta k} \mathrm{~d} k \\
& =e^{-r(T-t)} \int_{-\infty}^{+\infty} \exp [(\alpha+i \theta) k] \mathbb{E}\left[\left(\exp \left(Y_{t}\right)-\exp (k)\right)^{+} \mid \mathscr{F}_{t}\right] \mathrm{d} k, \\
\psi_{t}^{\alpha}(T, \theta) & =\frac{e^{-r(T-t)} \phi_{t}(T, \theta-(1+\alpha) i)}{(\alpha+i \theta)(\alpha+i \theta+1)} .
\end{aligned}
$$

The call price can be provided through inversion of Fourier transform given $\psi_{t}^{\alpha}(T, \theta)$ function having both odd imaginary and even real parts; so from equation (73), we have

$$
\begin{aligned}
\int_{-\infty}^{+\infty} C_{t}^{\alpha}(T, k) e^{i \theta k} \mathrm{~d} k & =\frac{e^{-r(T-t)} \phi_{t}(T, \theta-(1+\alpha) i)}{(\alpha+i \theta)(\alpha+i \theta+1)}, \\
C_{t}^{\alpha}(T, k) & =\frac{1}{2 \pi} \int_{-\infty}^{\infty} \psi_{t}^{\alpha}(T, \theta) e^{-i \theta k} \mathrm{~d} \theta
\end{aligned}
$$

Then, we have

$$
\begin{aligned}
& C_{t}(T, K)=\frac{e^{-\alpha k}}{2 \pi} \int_{-\infty}^{\infty} \psi_{t}^{\alpha}(T, \theta) e^{-i \theta k} \mathrm{~d} \theta, \\
& C_{t}(T, K)=\frac{e^{-\alpha k}}{2 \pi} \int_{-\infty}^{\infty} \frac{e^{-r(T-t)} \widetilde{\phi}_{t}(T, \theta-(1+\alpha) i)}{(\alpha+i \theta)(\alpha+i \theta+1)} e^{-i \theta k} \mathrm{~d} \theta .
\end{aligned}
$$

This is a Fourier transform: 


$$
C_{t}(T, K)=\frac{e^{-\alpha k}}{2 \pi} \operatorname{Re}\left(\int_{-\infty}^{\infty} \frac{e^{-r(T-t)} \widetilde{\phi}_{t}(T, \theta-(1+\alpha) i)}{(\alpha+i \theta)(\alpha+i \theta+1)} e^{-i \theta k} \mathrm{~d} \theta\right)
$$

Corollary 1. Let $D$ be the symmetric matrix, and it is sufficient to find the conditional characteristic function of the double Wishart $\Sigma_{t}$ and $\bar{\Sigma}_{t}$ given by (Appendix)

$$
\begin{aligned}
\tilde{\phi}_{\Sigma_{t} \bar{\Sigma}_{t}}^{D_{1} D_{2}} & =\mathbb{E}\left[\exp \left\{i \operatorname{Tr}\left[D_{1} \Sigma_{t}+D_{2} \bar{\Sigma}_{t}\right]\right\}\right] \\
& =\exp \left\{\operatorname{Tr}\left[A_{1}(\tau) \Sigma_{t}+A_{2}(\tau) \bar{\Sigma}_{t}\right]+C(\tau)\right\},
\end{aligned}
$$

where $A_{1}(\tau), A_{2}(\tau) \in M_{n}$, and $C \in \mathbb{C}$ verify the following dynamics:

$$
\begin{aligned}
& \left\{\begin{array}{l}
\frac{\mathrm{d} A_{1}(\tau)}{\mathrm{d} \tau}=A_{1}(\tau) M+M^{T} A_{1}(\tau)+2 A_{1}(\tau) Q^{T} Q A_{1}(\tau), \\
A_{1}(0)=i D_{1},
\end{array}\right. \\
& \left\{\begin{array}{l}
\frac{\mathrm{d} A_{2}(\tau)}{\mathrm{d} \tau}=A_{2}(\tau) \bar{M}+\bar{M}^{T} A_{2}(\tau)+2 A_{2}(\tau) \overline{\mathrm{Q}}^{T} \overline{\mathrm{Q}} A_{2}(\tau), \\
A_{2}(0)=i D_{2},
\end{array}\right. \\
& C(\tau)=\int_{0}^{\tau} \operatorname{Tr}\left[\beta Q^{T} \mathrm{Q} A_{1}(\tau) \mathrm{d} u+\bar{\beta} \bar{Q}^{T} \bar{Q} A_{2}(\tau) \mathrm{d} u\right] . \\
& \widetilde{\phi}_{\Sigma, \bar{\Sigma}}(t)=e^{\left\{\operatorname{Tr}\left[A_{1}(t) \Sigma_{0}+A_{2}(t) \bar{\Sigma}_{0}\right]+C(t)+\varepsilon(T-t)\right\},}, \\
& \quad \phi(\theta)=\frac{1}{(\alpha+i \theta)(\alpha+i \theta+1)} .
\end{aligned}
$$

Proposition 3. The call price under double Wishart is given as

$$
C_{t}(T, K)=\frac{e^{-\alpha k}}{2 \pi} \operatorname{Re}\left(\int_{-\infty}^{\infty} e^{-r(T-t)} \widetilde{\phi}_{\Sigma, \bar{\Sigma}}(t) \phi(\theta) e^{-i \theta k} \mathrm{~d} \theta\right),
$$

where
Proof. Let $\widetilde{\phi}(t, T)$ be the characteristic function of the logprice $Y_{t}$. We have,

$$
\begin{aligned}
Y_{t, T} & =\ln \left(\frac{X_{T}}{X_{t}}\right)=\ln \left(X_{T}\right)-\ln \left(X_{t}\right)=Y_{T}-Y_{t}, \\
\widetilde{\phi}_{\gamma, 0}(t, T) & =\mathbb{E}\left[\exp \left\{i \gamma Y_{t, T}\right\}\right] \\
& =\mathbb{E}\left[\left(\exp \left(-i \gamma Y_{t}\right)\right) \mathbb{E}_{t} \exp \left\{i \gamma Y_{T}\right\}\right] \\
& =\mathbb{E}\left[\exp \left(-i \gamma Y_{t}\right) \exp \left\{\operatorname{Tr}\left[\lambda_{1}(T-t) \Sigma_{t}+\lambda_{2}(T-t) \bar{\Sigma}_{t}\right]+i \gamma Y_{t}+\varepsilon(T-t)\right\}\right] \\
& =\exp \{\varepsilon(T-t)\} \mathbb{E}\left[\exp \left\{\operatorname{Tr}\left[\lambda_{1}(T-t) \Sigma_{t}+\lambda_{2}(T-t) \bar{\Sigma}_{t}\right]\right\}\right] \\
& =\exp \{\varepsilon(T-t)\} \exp \left\{\operatorname{Tr}\left[A_{1}(\tau) \Sigma_{0}+A_{2}(\tau) \bar{\Sigma}_{0}\right]+C(\tau)\right\} \\
& =\exp \{\varepsilon(T-t)\} \exp \left\{\operatorname{Tr}\left[A_{1}(t) \Sigma_{0}+A_{2}(t) \bar{\Sigma}_{0}\right]+C(t)\right\} \\
\widetilde{\phi}_{\gamma, 0}(t, T) & =\exp \left\{\operatorname{Tr}\left[A_{1}(t) \Sigma_{0}+A_{2}(t) \bar{\Sigma}_{0}\right]+C(t)+\varepsilon(T-t)\right\},
\end{aligned}
$$


where $A_{j}(t)$ is obtained from equation (79) with $\tau=t$ provided $A_{j}(0)=\lambda_{j}(T-t), j=1,2$. Hence,

$$
C_{t}(T, K)=\frac{e^{-\alpha k}}{2 \pi} \operatorname{Re}\left(\int_{-\infty}^{\infty} \frac{e^{-r(T-t)} e^{\left\{\operatorname{Tr}\left[A_{1}(t) \Sigma_{0}+A_{2}(t) \bar{\Sigma}_{0}\right]+C(t)+\varepsilon(T-t)\right\}}}{(\alpha+i \theta)(\alpha+i \theta+1)} e^{-i \theta k} \mathrm{~d} \theta\right)
$$

where

$$
\varepsilon(\tau)=\operatorname{Tr}\left[\log \left(\frac{1}{\sqrt{\left(H_{1}(\tau)\right)^{\beta}\left(I_{1}(\tau)\right)^{\bar{\beta}}}}\right)-\frac{1}{2}\left(\beta M^{T}+\bar{\beta} \bar{M}^{T}\right)+r \gamma\right] .
$$

3.4. Perturbation Techniques of the Riccati Differential Equations. This section deals with implementation of perturbation techniques since the system of the Riccati differential equations does not allow an analytical closed-form solution. This is due to noncommutativity of matrix multiplication, in order to approximate the call option price.

The method procedures retain the affine properties and higher orders comparable to the standard perturbation scheme on partial differential equations that is complex after the first order [9, 17]. Consider the differential Riccati equations of the double Wishart stochastic volatility model and take dimension $n=2$. The two characteristics orders in perturbation $p$ and $q$ are considered. The solution of $A(\tau)$ is given in the following form:

$$
A(\tau)=\sum_{i, j} p^{i / 2} q^{j / 2} A^{i, j}(\tau) .
$$

The developments in the perturbed differential equations are done by comparing the coefficients and identifying the terms in $p$ and $q$ to give expected approximations. Let $p=$ $M_{1}$ and $q=M_{2}$ are small while $v_{i}$ quantities remain constant. Then, we consider approximation at order one $(\sqrt{p}, \sqrt{q})$ and order two $(p, q)$ together with the following notations.

$$
\begin{aligned}
& M=\left(\begin{array}{cc}
-p & 0 \\
0 & -q
\end{array}\right)=-p\left(\begin{array}{ll}
1 & 0 \\
0 & 0
\end{array}\right)-q\left(\begin{array}{ll}
0 & 0 \\
0 & 1
\end{array}\right)=-p M_{1}-q M_{2}, \\
& \bar{M}=\left(\begin{array}{cc}
-\bar{p} & 0 \\
0 & -\bar{q}
\end{array}\right)=-\bar{p}\left(\begin{array}{ll}
1 & 0 \\
0 & 0
\end{array}\right)-\bar{q}\left(\begin{array}{ll}
0 & 0 \\
0 & 1
\end{array}\right)=-\bar{p} M_{1}-\bar{q} M_{2},
\end{aligned}
$$

and also noting that $Q$ is the volatility,

$$
\begin{aligned}
Q & =\sqrt{p} v_{1} M_{1}+\sqrt{q} v_{2} M_{2}, \\
Q^{2} & =p v_{1}^{2} M_{1}+q v_{2}^{2} M_{2},
\end{aligned}
$$

which can be written as

$$
Q=\sqrt{p} Q_{1}+\sqrt{q} Q_{2} .
$$

We can now rewrite the Riccati equations as in the form

$$
A_{1}^{\prime}(\tau)=p\left[-A_{1}(\tau) M_{1}-M_{1} A_{1}(\tau)+2 A_{1}(\tau) Q_{1}^{2} A_{1}(\tau)\right]+q\left[-A_{1}(\tau) M_{2}-M_{2} A_{1}(\tau)+2 A_{1}(\tau) Q_{2}^{2} A_{1}(\tau)\right]
$$

and for

$$
\begin{aligned}
\lambda_{1}^{\prime}(\tau)= & \frac{\gamma(\gamma-1)}{2} \mathbb{Q}_{2}+p\left(-\lambda_{1}(\tau) M_{1}-M_{1} \lambda_{1}(\tau)+2 \lambda_{1}(\tau) Q_{1}^{2} \lambda_{1}(\tau)\right)+2 \sqrt{p} \gamma R Q_{1} \lambda_{1}(\tau) \\
& +2 \sqrt{q} \gamma R Q_{2} \lambda_{1}(\tau)+q\left(-\lambda_{1}(\tau) M_{2}-M_{2} \lambda_{1}(\tau)+2 \lambda_{1}(\tau) Q_{2}^{2} \lambda_{1}(\tau)\right), \\
\lambda_{2}^{\prime}(\tau)= & \frac{\gamma(\gamma-1)}{2} \mathbb{1}_{2}+\bar{p}\left(-\lambda_{2}(\tau) M_{1}-M_{1} \lambda_{2}(\tau)+2 \lambda_{2}(\tau) \bar{Q}_{1}^{2} \lambda_{2}(\tau)\right)+2 \sqrt{\bar{p}} \gamma \bar{R} \bar{Q}_{1} \lambda_{2}(\tau) \\
& +2 \sqrt{\bar{q}} \gamma \bar{R} \bar{Q}_{2} \lambda_{2}(\tau)+\bar{q}\left(-\lambda_{2}(\tau) M_{2}-M_{2} \lambda_{2}(\tau)+2 \lambda_{2}(\tau) \bar{Q}_{2}^{2} \lambda_{2}(\tau)\right) .
\end{aligned}
$$


Development of the Riccati functions: by dealing with the development of the Riccati functions $A_{1}, A_{2}, \lambda_{1}, \lambda_{2}, C$, and $\varepsilon$, the perturbation of the Riccati equations at order two (order 2) is considered to obtain the European call option pricing formula as follows:

$$
A_{k}(\tau)=A_{k}^{0,0}(\tau)+\sqrt{p} A_{k}^{1,0}(\tau)+\sqrt{q} A_{k}^{0,1}(\tau)+p A_{k}^{2,0}(\tau)+q A_{k}^{0,2}(\tau)+\sqrt{p q} A_{k}^{1,1}(\tau)+o(\max (p, q)), \quad k=1,2
$$

Let us determine $A_{k}^{0,0}, \ldots, A_{k}^{1,1}$.

By identifying the terms in respective orders, we have

$$
\left(A_{1}^{0,0}(\tau)\right)^{\prime}=0, \quad A_{1}^{0,0}(\tau) \in M_{2}(\mathbb{C}) .
$$

$$
D_{1}=\left(\begin{array}{cc}
d_{1} & 0 \\
0 & d_{1}
\end{array}\right)
$$

Since $A_{1}(0)=i D_{1}$,

Then, we obtain

$$
A_{1}^{0,0}(0)+\sqrt{p} A_{1}^{1,0}(0)+\sqrt{q} A_{1}^{0,1}(0)+p A_{1}^{2,0}(0)+q A_{1}^{0,2}(0)+\sqrt{p q} A_{1}^{1,1}(0)=i D_{1} .
$$

Then,

$$
\begin{aligned}
A_{1}^{0,0}(0) & =i D_{1}=i d_{1} \rrbracket_{2}, \\
A_{1}^{1,0}(0) & =A_{1}^{0,1}(0)=A_{1}^{2,0}(0)=A_{1}^{0,2}(0)=A_{1}^{1,1}(0)=(0), \\
A_{1}^{2,0}(\tau) & =-2 d_{1}\left(i+d_{1} v_{1}^{2}\right) \tau M_{1}, \\
A_{1}^{0,2}(\tau) & =-2 d_{1}\left(i+d_{1} v_{2}^{2}\right) \tau M_{2}, \\
\left(A_{1}^{1,1}(\tau)\right)^{\prime} & =0, \Rightarrow A_{1}^{1,1}(\tau)=C,
\end{aligned}
$$

$$
\begin{gathered}
A_{2}^{\prime}(\tau)=\bar{p}\left[-A_{2}(\tau) M_{1}-M_{1} A_{2}(\tau)+2 A_{2}(\tau) \bar{Q}_{1}^{2} A_{2}(\tau)\right]+\bar{q}\left[-A_{2}(\tau) M_{2}-M_{2} A_{2}(\tau)+2 A_{2}(\tau) \bar{Q}_{2}^{2} A_{2}(\tau)\right], \\
A_{2}^{\prime}(\tau)=\left(A_{2}^{0,0}(\tau)\right)^{\prime}+\sqrt{\bar{p}}\left(A_{2}^{1,0}(\tau)\right)^{\prime}+\sqrt{\bar{q}}\left(A_{2}^{0,1}(\tau)\right)^{\prime}+\bar{p}\left(A_{2}^{2,0}(\tau)\right)^{\prime}+\bar{q}\left(A_{2}^{0,2}(\tau)\right)^{\prime}+\sqrt{\bar{p} \bar{q}}\left(A_{2}^{1,1}(\tau)\right)^{\prime}, \\
D_{2}=\left(\begin{array}{cc}
d_{2} & 0 \\
0 & d_{2}
\end{array}\right) .
\end{gathered}
$$$$
\text { since } A_{1}^{1,1}(0)=0 \text {, for all } \tau \in \mathbb{R}_{+}, C=0 \text {. }
$$

For function $A_{2}(\tau)$, we go through the same procedures:

then,

$$
\left(A_{2}^{0,0}(\tau)\right)^{\prime}=0, \quad \Rightarrow A_{2}^{0,0}(\tau) \in M_{2}(\mathbb{C}) .
$$

Since $A_{2}(0)=i D_{2}$,

$$
\begin{aligned}
& \left(A_{2}^{0,0}(0)\right)^{\prime}+\sqrt{\bar{p}}\left(A_{2}^{1,0}(0)\right)^{\prime}+\sqrt{\bar{q}}\left(A_{2}^{0,1}(0)\right)^{\prime}+\bar{p}\left(A_{2}^{2,0}(0)\right)^{\prime}+\bar{q}\left(A_{2}^{0,2}(0)\right)^{\prime}+\sqrt{\bar{p} \bar{q}}\left(A_{2}^{1,1}(0)\right)^{\prime}=i D_{2}, \\
& A_{2}^{0,0}(0)=i D_{2}=i d_{2} \rrbracket_{2} \\
& A_{2}^{1,0}(0)=A_{2}^{0,1}(0)=A_{2}^{2,0}(0)=A_{2}^{0,2}(0)=A_{2}^{1,1}(0)=(0) .
\end{aligned}
$$

We proceed by obtaining

$$
\begin{aligned}
& A_{2}^{2,0}(\tau)=-2 d_{2}\left(i+d_{2} \bar{v}_{1}^{2}\right) \tau M_{1}, \\
& A_{2}^{0,2}(\tau)=-2 d_{2}\left(i+d_{2} \bar{v}_{2}^{2}\right) \tau M_{2},
\end{aligned}
$$

and $\left(A_{2}^{1,1}(\tau)\right)^{\prime}=0, A_{2}^{1,1}(\tau)=0$; since $A_{2}^{1,1}(0)=0$, for all $\tau \in \mathbb{R}_{+}, C=0$ is constant. So now, we can get the functions of $\lambda_{1}$ and $\lambda_{2}$ from the ordinary differential equations (90) and (91) as follows: 


$$
\begin{aligned}
\lambda_{1}^{\prime}(\tau)= & \frac{\gamma(\gamma-1)}{2} \mathbb{1}_{2}+p\left(-\lambda_{1}(\tau) M_{1}-M_{1} \lambda_{1}(\tau)+2 \lambda_{1}(\tau) Q_{1}^{2} \lambda_{1}(\tau)\right)+2 \sqrt{p} \gamma R Q_{1} \lambda_{1}(\tau) \\
& +2 \sqrt{q} \gamma R Q_{2} \lambda_{1}(\tau)+q\left(-\lambda_{1}(\tau) M_{2}-M_{2} \lambda_{1}(\tau)+2 \lambda_{1}(\tau) Q_{2}^{2} \lambda_{1}(\tau)\right), \\
\lambda_{1}(\tau)= & \lambda_{1}^{0,0}(\tau)+\sqrt{p} \lambda_{1}^{1,0}(\tau)+\sqrt{q} \lambda_{1}^{0,1}(\tau)+p \lambda_{1}^{2,0}(\tau)+q \lambda_{1}^{0,2}(\tau)+\sqrt{p q} \lambda_{1}^{1,1}(\tau)+o(\max (p, q)),
\end{aligned}
$$

such that

$$
\begin{aligned}
& \lambda_{1}^{0,0}(\tau)=\frac{\gamma(\gamma-1)}{2} \tau \rrbracket_{2}, \\
& \lambda_{1}^{1,0}(\tau)=\frac{\gamma^{2}(\gamma-1)}{2} v_{1} \tau^{2}\left(R M_{1}\right), \\
& \lambda_{1}^{0,1}(\tau)=\frac{\gamma^{2}(\gamma-1)}{2} v_{2} \tau^{2}\left(R M_{2}\right), \\
& \lambda_{1}^{2,0}(\tau)=-\frac{\gamma(\gamma-1)}{2} \tau^{2} M_{1}+\frac{\gamma^{2}(\gamma-1)^{2}}{6} \nu_{1}^{2} \tau^{3} M_{1}+\frac{\gamma^{3}(\gamma-1)}{3} \nu_{1}^{2} \tau^{3}\left(R M_{1}\right)^{2}, \\
& \lambda_{1}^{0,2}(\tau)=-\frac{\gamma(\gamma-1)}{2} \tau^{2} M_{2}+\frac{\gamma^{2}(\gamma-1)}{6} v_{2}^{2} \tau^{3} M_{2}+\frac{\gamma^{3}(\gamma-1)}{3} v_{2}^{2} \tau^{3}\left(R M_{2}\right)^{2}, \\
& \lambda_{1}^{1,1}(\tau)=\frac{\gamma^{3}(\gamma-1)}{3} \nu_{1} \nu_{2} \tau^{3}\left[\left(R M_{1}\right)\left(R M_{2}\right)+\left(R M_{2}\right)\left(R M_{1}\right)\right] .
\end{aligned}
$$

Then, similar for $\lambda_{2}$, we have the following approximations:

$$
\begin{aligned}
& \lambda_{2}^{0,0}(\tau)=\frac{\gamma(\gamma-1)}{2} \tau \rrbracket_{2}, \\
& \lambda_{2}^{1,0}(\tau)=\frac{\gamma^{2}(\gamma-1)}{2} \bar{v}_{1} \tau^{2}\left(\bar{R} M_{1}\right), \\
& \lambda_{2}^{0,1}(\tau)=\frac{\gamma^{2}(\gamma-1)}{2} \bar{v}_{2} \tau^{2}\left(\bar{R} M_{2}\right), \\
& \lambda_{2}^{2,0}(\tau)=-\frac{\gamma(\gamma-1)}{2} \tau^{2} M_{1}+\frac{\gamma^{2}(\gamma-1)^{2}}{6} \bar{\nu}_{1}^{2} \tau^{3} M_{1}+\frac{\gamma^{3}(\gamma-1)}{3} \bar{\nu}_{1}^{2} \tau^{3}\left(\bar{R} M_{1}\right)^{2}, \\
& \lambda_{2}^{0,2}(\tau)=-\frac{\gamma(\gamma-1)}{2} \tau^{2} M_{2}+\frac{\gamma^{2}(\gamma-1)}{6} \bar{v}_{2}^{2} \tau^{3} M_{2}+\frac{\gamma^{3}(\gamma-1)}{3} \bar{\nu}_{2}^{2} \tau^{3}\left(\bar{R} M_{2}\right)^{2}, \\
& \lambda_{2}^{1,1}(\tau)=\frac{\gamma^{3}(\gamma-1)}{3} \bar{\nu}_{1} \bar{\nu}_{2} \tau^{3}\left[\left(\bar{R} M_{1}\right)\left(\bar{R} M_{2}\right)+\left(\bar{R} M_{2}\right)\left(\bar{R} M_{1}\right)\right] .
\end{aligned}
$$


Then, we look at the Riccati differential equation for $C(\tau)$ :

$$
\begin{aligned}
C^{\prime}(\tau) & =\beta \operatorname{Tr}\left[p v_{1}^{2} M_{1} A_{1}(\tau)+q v_{2}^{2} M_{2} A_{1}(\tau)\right]+\bar{\beta} \operatorname{Tr}\left[p \bar{v}_{1}^{2} M_{1} A_{2}(\tau)+q \bar{\nu}_{2}^{2} M_{2} A_{2}(\tau)\right], \\
C^{0,0}(\tau) & =0 .
\end{aligned}
$$

Since $C(0)=0$,

For the $\varepsilon$ differential equation,

$$
\begin{aligned}
& C^{1,0}(\tau)=C^{0,1}(\tau)=0, \\
& C^{2,0}(\tau)=i \beta v_{1}^{2} d_{1} \tau+i \bar{\beta} \bar{\nu}_{1}^{2} d_{2} \tau, \\
& C^{0,2}(\tau)=i \beta v_{2}^{2} d_{1} \tau+i \bar{\beta} \bar{\nu}_{2}^{2} d_{2} \tau, \\
& C^{1,1}(\tau)=0 .
\end{aligned}
$$

$$
\varepsilon^{\prime}(\tau)=r \gamma+\beta \operatorname{Tr}\left[p v_{1}^{2} M_{1} \lambda_{1}(\tau)+q v_{2}^{2} M_{2} \lambda_{1}(\tau)\right]+\bar{\beta} \operatorname{Tr}\left[p \bar{\nu}_{1}^{2} M_{1} \lambda_{2}(\tau)+q \bar{\nu}_{2}^{2} M_{2} \lambda_{2}(\tau)\right]
$$

we have,

$$
\begin{aligned}
& \mathcal{E}^{0,0}(\tau)=r \gamma \tau, \\
& \varepsilon^{0,1}(\tau)=0, \\
& \varepsilon^{2,0}(\tau)=\beta v_{1}^{2} \frac{\gamma(\gamma-1)}{4} \tau^{2}+\bar{\beta} \bar{\nu}_{1}^{2} \frac{\gamma(\gamma-1)}{4} \tau^{2}, \\
& \varepsilon^{0,2}(\tau)=\beta v_{2}^{2} \frac{\gamma(\gamma-1)}{4} \tau^{2}+\bar{\beta} \bar{\nu}_{2}^{2} \frac{\gamma(\gamma-1)}{4} \tau^{2}, \\
& \varepsilon^{1,1}(\tau)=0 .
\end{aligned}
$$

$$
\begin{aligned}
& A_{1}(\tau)=A_{1}^{0,0}(\tau)+\sqrt{p} A_{1}^{1,0}(\tau)+\sqrt{q} A_{1}^{0,1}(\tau)+p A_{1}^{2,0}(\tau)+q A_{1}^{0,2}(\tau)+\sqrt{p q} A_{1}^{1,1}(\tau)+o(\max (p, q)) \\
& A_{1}(\tau)=\left(\begin{array}{cc}
i d_{1} & 0 \\
0 & i d_{1}
\end{array}\right)+\left(\begin{array}{cc}
-2 p d_{1}\left(i+d_{1} v_{1}^{2}\right) \tau & 0 \\
0 & 0
\end{array}\right)+\left(\begin{array}{cc}
0 & 0 \\
0 & -2 q d_{1}\left(i+d_{1} v_{2}^{2}\right) \tau
\end{array}\right)+o(\max (p, q)) \\
& A_{1}(\tau)=\left(\begin{array}{cc}
-2 p d_{1}^{2} v_{1}^{2} \tau+i d_{1}(1-2 p \tau) & 0 \\
0 & -2 q d_{1}^{2} v_{2}^{2} \tau+i d_{1}(1-2 q \tau)
\end{array}\right) \\
& A_{2}(\tau)=\left(\begin{array}{cc}
-2 p d_{2}^{2} \bar{v}_{1}^{2} \tau+i d_{2}(1-2 p \tau) & 0 \\
0 & -2 q d_{2}^{2} \bar{v}_{2}^{2} \tau+i d_{2}(1-2 q \tau)
\end{array}\right)
\end{aligned}
$$


The variance processes $\Sigma_{0}$ and $\bar{\Sigma}_{0}$ are given as

$$
\begin{aligned}
\Sigma_{0} & =\left(\begin{array}{cc}
u & v \\
v & w
\end{array}\right), \\
\bar{\Sigma}_{0} & =\left(\begin{array}{cc}
\bar{u} & \bar{v} \\
\bar{v} & \bar{w}
\end{array}\right), \\
A_{1}(\tau) \Sigma_{0}= & \left(\begin{array}{cc}
-2 p d_{1}^{2} v_{1}^{2} \tau+i d_{1}(1-2 p \tau) & 0 \\
0 & -2 q d_{1}^{2} v_{2}^{2} \tau+i d_{1}(1-2 q \tau)
\end{array}\right)\left(\begin{array}{cc}
u & v \\
v & w
\end{array}\right), \\
A_{1}(\tau) \Sigma_{0}= & \left(\begin{array}{cc}
-2 u p d_{1}^{2} v_{1}^{2} \tau+i d_{1}(1-2 p \tau) u & -2 v p d_{1}^{2} v_{1}^{2} \tau+i d_{1}(1-2 p \tau) v \\
-2 q v d_{1}^{2} v_{2}^{2} \tau+i d_{1}(1-2 q \tau) v & -2 q w d_{1}^{2} v_{2}^{2} \tau+i d_{1}(1-2 q \tau) w
\end{array}\right), \\
A_{2}(\tau) \bar{\Sigma}_{0}= & \left(\begin{array}{cc}
-2 \bar{u} p d_{2}^{2} \bar{\nu}_{1}^{2} \tau+i d_{2}(1-2 p \tau) \bar{u} & -2 p \bar{v} d_{2}^{2} \bar{v}_{1}^{2} \tau+i d_{2}(1-2 p \tau) \bar{v} \\
-2 q \bar{v} d_{2}^{2} \bar{v}_{2}^{2} \tau+i d_{2}(1-2 q \tau) \bar{v} & -2 q \bar{w} d_{2}^{2} \bar{v}_{2}^{2} \tau+i d_{2}(1-2 q \tau) \bar{w}
\end{array}\right) .
\end{aligned}
$$

Taking the trace,

$$
\begin{aligned}
& \operatorname{Tr}\left(A_{1}(\tau) \Sigma_{0}\right)=-2 d_{1}^{2}\left[u p v_{1}^{2}+w q v_{2}^{2}\right] \tau+i[(1-2 p \tau) u+(1-2 q \tau) w] d_{1}, \\
& \operatorname{Tr}\left(A_{2}(\tau) \bar{\Sigma}_{0}\right)=-2 d_{2}^{2}\left[\bar{u} p \bar{v}_{1}^{2}+\bar{w} q \bar{v}_{2}^{2}\right] \tau+i[(1-2 p \tau) \bar{u}+(1-2 q \tau) \bar{w}] d_{2} .
\end{aligned}
$$

For functions $C$ and $\varepsilon$,

$$
\begin{aligned}
C(\tau) & =p C^{2,0}(\tau)+q C^{0,2}(\tau)+o(\max (p, q)) \\
& =p\left(i \beta v_{1}^{2} d_{1} \tau+i \bar{\beta} \bar{\nu}_{1}^{2} d_{2} \tau\right)+q\left(i \beta v_{2}^{2} d_{1} \tau+i \bar{\beta} \bar{\nu}_{2}^{2} d_{2} \tau\right)+o(\max (p, q)) \\
& =i \beta d_{1}\left(p v_{1}^{2}+q v_{2}^{2}\right) \tau+i \bar{\beta} d_{2}\left(p \bar{\nu}_{1}^{2}+q \bar{\nu}_{2}^{2}\right) \tau+o(\max (p, q)) .
\end{aligned}
$$

Then,

$$
\begin{aligned}
\varepsilon(\tau) & =\varepsilon^{0,0}(\tau)+p \varepsilon^{2,0}(\tau)+q \varepsilon^{0,2}(\tau)+o(\max (p, q)) \\
& =r \gamma \tau+\frac{\gamma(\gamma-1)}{4} \beta\left(p v_{1}^{2}+q \nu_{2}^{2}\right) \tau^{2}+\frac{\gamma(\gamma-1)}{4} \bar{\beta}\left(p \bar{\nu}_{1}^{2}+q \bar{\nu}_{2}^{2}\right) \tau^{2}+o(\max (p, q))
\end{aligned}
$$

Substituting back in equation (80), we have 


$$
\begin{aligned}
\widetilde{\phi}_{\Sigma \bar{\Sigma}}(\tau)= & \exp \left\{r \gamma(T-t)+p\left[-2 d_{1}^{2} u v_{1}^{2} t-2 d_{2}^{2} \bar{u} \bar{v}_{1}^{2} t+\frac{\gamma(\gamma-1)}{4} \beta v_{1}^{2}(T-t)^{2}+\frac{\gamma(\gamma-1)}{\beta} \bar{\nu}_{1}^{2}(T-t)^{2}\right]\right. \\
& +q\left[-2 d_{1}^{2} w v_{2}^{2} t-2 d_{2}^{2} \bar{w} v_{2}^{2} t+\frac{\gamma(\gamma-1)}{4} \beta v_{2}^{2}(T-t)^{2}+\frac{\gamma(\gamma-1)}{4} \bar{\beta}_{2}^{2}(T-t)^{2}\right] \\
& \times \exp \left\{i \left[(1-2 p t) u d_{1}+(1-2 q t) w d_{1}+(1-2 p t) \bar{u} d_{2}\right.\right. \\
& +(1-2 q t) \bar{w} d_{2}+\beta d_{1}\left(p v_{1}^{2}+q v_{2}^{2}\right) t+\bar{\beta} d_{2}\left(p \bar{v}_{1}^{2}+q \bar{v}_{2}^{2}\right) t+o(\max (p, q)) .
\end{aligned}
$$

This can still be written as

where

$$
\widetilde{\phi}_{\Sigma \bar{\Sigma}}(\tau)=e^{\Delta_{1}(t)} e^{i \Delta_{2}(t)}
$$

$$
\begin{aligned}
\Delta_{1}(t)= & \left\{r \gamma(T-t)+p\left[-2 d_{1}^{2} u v_{1}^{2} t-2 d_{2}^{2} \bar{u}_{1}^{2} t+\frac{\gamma(\gamma-1)}{4} \beta v_{1}^{2}(T-t)^{2}+\frac{\gamma(\gamma-1)}{4} \bar{\beta} \bar{\nu}_{1}^{2}(T-t)^{2}\right]\right. \\
& +q\left[-2 d_{1}^{2} w v_{2}^{2} t-2 d_{2}^{2} \bar{w} \bar{v}_{2}^{2} t+\frac{\gamma(\gamma-1)}{4} \beta v_{2}^{2}(T-t)^{2}+\frac{\gamma(\gamma-1)}{4} \bar{\beta} \bar{v}_{2}^{2}(T-t)^{2}\right], \\
\Delta_{2}(t)= & \left\{(1-2 p t) u d_{1}+(1-2 q t) w d_{1}+(1-2 p t) \bar{u} d_{2}\right. \\
& +(1-2 q t) \bar{w} d_{2}+\beta d_{1}\left(p v_{1}^{2}+q v_{2}^{2}\right) t+\bar{\beta} d_{2}\left(p \bar{v}_{1}^{2}+q \bar{v}_{2}^{2}\right) t+o(\max (p, q)),
\end{aligned}
$$

such that the call price is as follows:

$$
\begin{aligned}
C_{t}(T, K) & =\frac{e^{-\alpha k}}{2 \pi} \operatorname{Re}\left(\int_{-\infty}^{\infty} e^{-r(T-t)} \widetilde{\phi}_{\Sigma \bar{\Sigma}}(t) \phi(\theta) e^{-i \theta k} \mathrm{~d} \theta\right) \\
& \approx \frac{e^{-\alpha k}}{2 \pi} \operatorname{Re}\left(\int_{-\infty}^{\infty} \frac{e^{-r(T-t)} e^{\Delta_{1}(t)} e^{i \Delta_{2}(t)} e^{-i \theta k}}{(\alpha+i \theta)(\alpha+1+i \theta)} \mathrm{d} \theta\right)
\end{aligned}
$$

since

$$
\begin{aligned}
\int_{-\infty}^{\infty} \frac{e^{-r(T-t)} e^{\Delta_{1}(t)} e^{i \Delta_{2}(t)} e^{-i \theta k}}{(\alpha+i \theta)(\alpha+1+i \theta)} \mathrm{d} \theta & =e^{-r(T-t)} e^{\Delta_{1}(t)} e^{i \Delta_{2}(t)} \int_{-\infty}^{\infty} \frac{e^{-i \theta k}}{(\alpha+i \theta)(\alpha+1+i \theta)} \mathrm{d} \theta \\
& =e^{-r(T-t)} e^{\Delta_{1}(t)} e^{i \Delta_{2}(t)}\left[2 \pi\left(e^{\alpha k}-e^{k(\alpha+1)}\right)\right] .
\end{aligned}
$$

Finally, we obtain the approximated European call option price as follows:

$$
C_{t}(T, K) \approx e^{\left(-r(T-t)+\Delta_{1}(t)\right)}\left(1-e^{k}\right) \cos \Delta_{2}(t) .
$$

\section{Numerical Illustration}

In this section, we deal with illustrative numerical examples to examine the implications of volatility in option pricing under the double Wishart stochastic volatility model. 


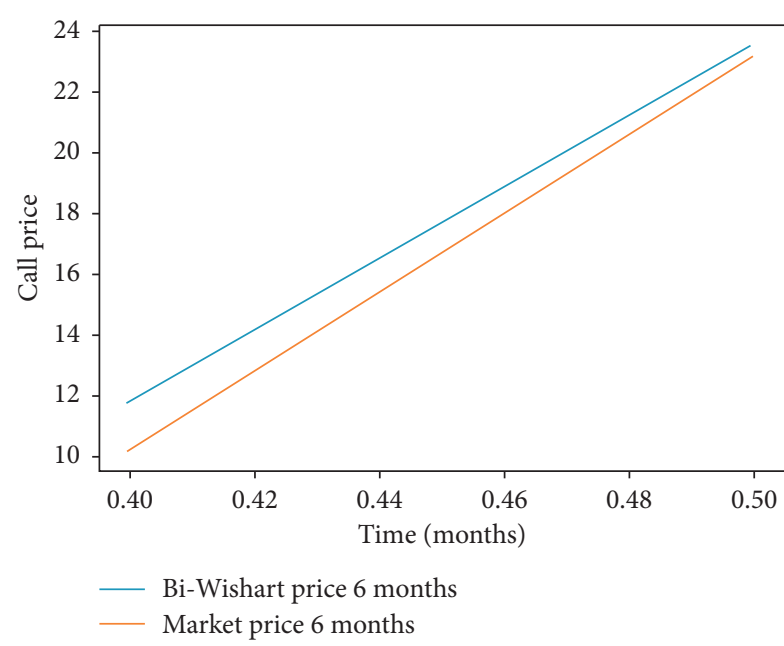

FIgURE 1: The price predictions at 6 months maturity time and the effect of model parameters on the call option price.

\subsection{Comparison between the Double Wishart Model Price and} the Market Price. The volatility specification of the double Wishart model remarkably makes it flexible to produce price predictions which exhibit a similar price behavior with the market price. We quote market data drawn from QQQ (a fund by Invesco that tracks the performance of the stocks listed under the NASDAQ Index) options, April 2020.

Let us consider the estimated parameter values from the market data; the variance matrices $\Sigma$ and $\bar{\Sigma}$, with volatility of volatility matrices $Q$ and $\bar{Q}$, are considered since they are very important parameters in a stochastic volatility model.

$$
\begin{aligned}
& \Sigma_{0}=\left(\begin{array}{cc}
0.00008 & 0 \\
0 & 0.000095
\end{array}\right), \\
& \bar{\Sigma}_{0}=\left(\begin{array}{cc}
0.00012 & 0 \\
0 & 0.0001
\end{array}\right), \\
& Q=\left(\begin{array}{cc}
0.10 & 0 \\
0 & 0.23
\end{array}\right), \\
& \bar{Q}=\left(\begin{array}{cc}
0.20 & 0 \\
0 & 0.25
\end{array}\right) .
\end{aligned}
$$

While, the strike price is $K=189$ and interest rate is $r=0.05$.

Figure 1 illustrates the effect of implied volatilities on the call price under the double Wishart model. Given the parameter values, we observe that the price predictions under the double Wishart model exhibits the same behavior with respect to the market price in short maturity time. This depends also on the choice of the model parameters, such as $\gamma=0.5, \beta=4, \bar{\beta}=3, d_{1}=0.45$, and $d_{2}=0.479$. This example proves that the double Wishart volatility model has greater flexibility.

Figure 2 shows that a slight change in model parameters leads to the changes in the model call option price predictions. We observe that as time tends to maturity time of six months, $\left(d_{2}=0.478\right)$, the model price movement behaves almost the same with the market price.

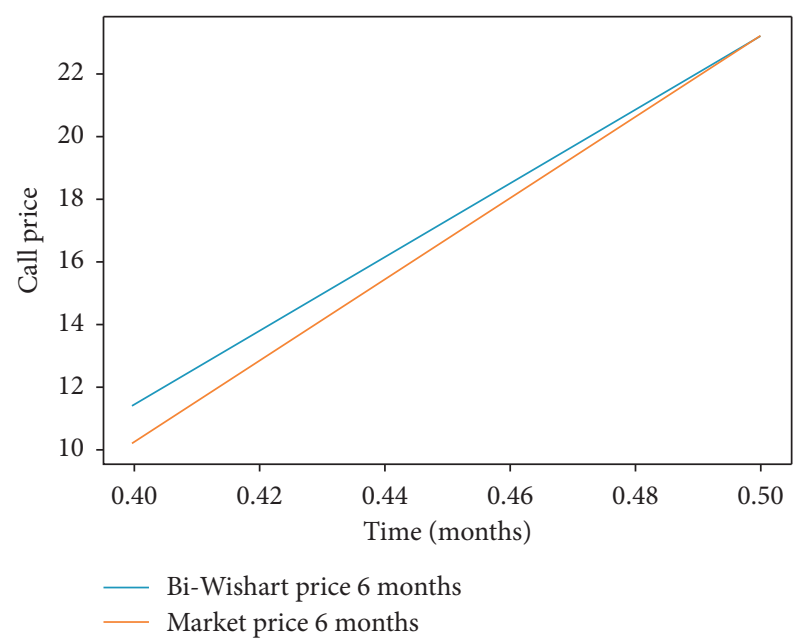

FIgURE 2: The call price predictions under the double Wishart model and market prices at 6 months maturity time.

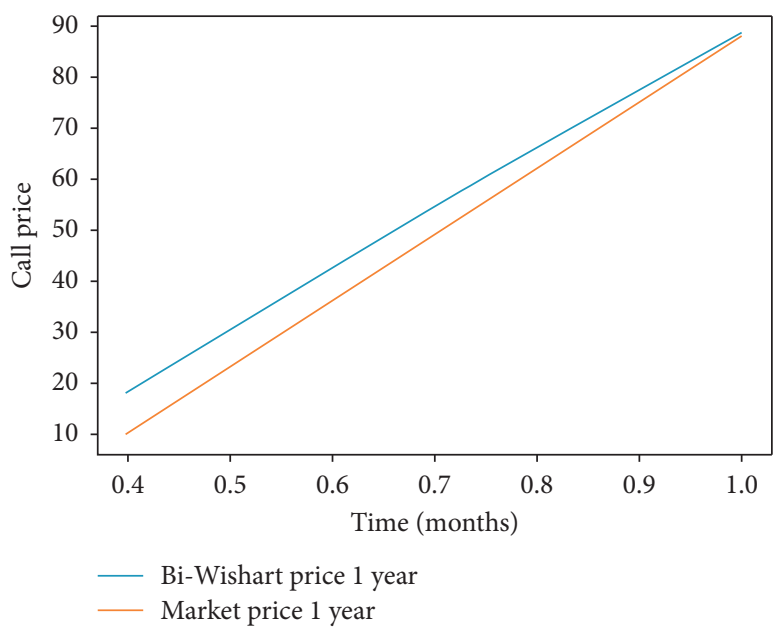

FIGURE 3: The double Wishart model price predictions and market price at 1 year maturity time.

The parameters $\gamma=0.5, \beta=4, \bar{\beta}=3$, and $d_{1}=0.45$ are maintained.

Figure 3 demonstrates the effect of parameters on the call European option price under the double Wishart model. The parameters $\gamma=1, \beta=4, \bar{\beta}=3, d_{1}=0.45$, and $d_{2}=0.4968$. The model shows price predictions exhibiting the same market price behavior, in long maturity time of 1 year. We note that the model parameters influence the prices greatly and help long position holders not to experience arbitrage profits.

\section{Conclusion}

The generalization of the Heston model into a multifactor form with two dependence matrices efficiently solves the problem of pricing European call option and financial market data fitting for short or long maturities. The new Wishart affine model retains the remarkable property of analytical tractability, and this allows the model to form a closed-form solution of the conditional characteristic 
function for the call option price expression, which is obtained through Fourier transforms together with perturbation methods.

The numerical results show that the double Wishart stochastic volatility model produces price predictions similar to the market prices. Notably the effect of parameters on our model makes it flexible enough for short or long maturities. We recommend future work on discretization schemes and also to investigate the behavior of nondiagonal matrix components in the model.

\section{Appendix}

\section{A. Change of Probability Measure}

From mathematics perspective, it is important to discuss the change of the probability measure to allow a change of the drift in the process dynamics. In the financial application, more so in the practical aspects, the Wishart process has to be simulated in its general form with $\beta \geq n+1$, such that $\beta \in \mathbb{R}$. The function allows to write $K=\beta+2 \lambda$ with $\beta=$ $K \geq n+1$ and $\lambda$ real number, such that $0 \leq \lambda \leq 1 / 2$.

The objective of this idea is to find a change of the probability measure in order to change the generalized Wishart diffusion into the simple one, where $\beta$ is an integer. Therefore, the new probability measure $\mathbb{P}$, following Benabid et al. [9] and Shreve [19], can be expressed as follows.

Theorem 1. Let $(\Omega, \mathscr{F}, \mathbb{Q})$ be the probability space equipped with filtration $\mathscr{F}_{t}$ satisfying usual conditions.

Let $q=\beta+\lambda-n-1$. If $h_{T}(\mathbb{Q}, \mathbb{P})=(\mathrm{d} \mathbb{Q} / \mathrm{d} \mathbb{P})$ defines the Radon-Nikodym derivative of $\mathbb{Q}$ with respect to $\mathbb{P}$, then

$$
h_{T}(\mathbb{Q}, \mathbb{P})=\left(\frac{\operatorname{det}\left(\Sigma_{T}\right)}{\operatorname{det}\left(\Sigma_{0}\right)}\right)^{\lambda / 2} \exp (-\lambda T \operatorname{Tr}(M)) \exp \left[\frac{-\lambda}{2} q \int_{0}^{T} \operatorname{Tr}\left(\Sigma_{s}^{-1} Q^{T} Q\right) \mathrm{d} s\right]
$$

Proof. The probability measure $\mathbb{P}$ can be specified through an exponential martingale [9]:

$$
\frac{\mathrm{d} \mathbb{Q}}{\mathrm{dP}}=\exp \left\{\lambda \int_{0}^{T} \operatorname{Tr}\left[\sqrt{\Sigma_{t}^{-1}} \mathrm{~d} W_{s} Q\right]-\frac{\lambda^{2}}{2} \int_{0}^{T} \operatorname{Tr}\left(\Sigma_{s}^{-1} Q^{T} Q\right) \mathrm{d} s\right\} .
$$

We can define a new process as

$$
W_{t}=\widetilde{W_{t}}+\lambda \int_{0}^{t} \sqrt{\Sigma_{s}^{-1}} Q^{T} \mathrm{~d} s
$$

It is simple to check by Girsanov theorem that $W$ is a matrix-valued Brownian motion under the probability measure $\mathbb{P}$. Consequently, the dynamics of the Wishart process under the probability measure $\mathbb{P}$ can be written as follows:

$$
\mathrm{d} \Sigma_{t}=\left(\beta Q Q^{T}+M \Sigma_{t}+\Sigma_{t} M^{T}\right) \mathrm{d} t+\sqrt{\Sigma_{t}} \mathrm{~d} W_{t} Q+Q^{T} \mathrm{~d} W_{t}^{T} \sqrt{\Sigma_{t}},
$$

where

$$
\begin{aligned}
W_{t} & =\widetilde{W}_{t}+\lambda \int_{0}^{t} \sqrt{\Sigma_{s}^{-1}} Q^{T} \mathrm{~d} s, \\
\mathrm{~d} W_{t} & =\mathrm{d} \widetilde{W}_{t}+\lambda \sqrt{\Sigma_{s}^{-1}} Q^{T} \mathrm{~d} t .
\end{aligned}
$$

Replacing $W_{t}$ in the Wishart dynamic process,

$$
\begin{aligned}
& \mathrm{d} \Sigma_{t}=\left(\beta Q Q^{T}+M \Sigma_{t}+\Sigma_{t} M^{T}\right) \mathrm{d} t+\sqrt{\Sigma_{t}}\left(\mathrm{~d} \widetilde{W_{t}}+\lambda \sqrt{\Sigma_{s}^{-1}} Q^{T} \mathrm{~d} t\right) Q+Q^{T}\left(\mathrm{~d} \widetilde{W}_{t}+\lambda \sqrt{\Sigma_{s}^{-1}} Q^{T} \mathrm{~d} t\right)^{T} \sqrt{\Sigma_{t}}, \\
& \mathrm{~d} \Sigma_{t}=\left(\beta Q Q^{T}+M \Sigma_{t}+\Sigma_{t} M^{T}\right) \mathrm{d} t+\sqrt{\Sigma_{t}} \mathrm{~d} \widetilde{W}_{t} Q+Q^{T} \mathrm{~d} \widetilde{W}_{t}^{T} \sqrt{\Sigma_{t}}+\lambda Q^{T} Q \mathrm{~d} t+\lambda Q^{T} Q \mathrm{~d} t .
\end{aligned}
$$

Then,

$$
\mathrm{d} \Sigma_{t}=\left((\beta+2 \lambda) Q Q^{T}+M \Sigma_{t}+\Sigma_{t} M^{T}\right) \mathrm{d} t+\sqrt{\Sigma_{t}} \mathrm{~d} \widetilde{W_{t}} Q+Q^{T} \mathrm{~d} \widetilde{W}_{t}^{T} \sqrt{\Sigma_{t}} .
$$

The Radon-Nikodym derivative can be handled using the determinant dynamics. 


$$
\begin{aligned}
\int_{0}^{T} \operatorname{dlog}\left(\operatorname{det} \Sigma_{s}\right) & =\int_{0}^{T}\left[(\beta-n-1) \operatorname{Tr}\left(\Sigma_{s}^{-1} Q^{T} Q\right)+2 \operatorname{Tr}(M)\right] \mathrm{d} s+2 \int_{0}^{T} \operatorname{Tr}\left[\sqrt{\Sigma_{s}^{-1}} \mathrm{~d} W_{s} Q\right] \\
\log \left(\frac{\operatorname{det} \Sigma_{T}}{\operatorname{det} \Sigma_{0}}\right) & =2 \operatorname{Tr}(M)+\int_{0}^{T}(\beta-n-1) \operatorname{Tr}\left(\Sigma_{s}^{-1} Q^{T} Q\right) \mathrm{d} s+2 \int_{0}^{T} \operatorname{Tr}\left[\sqrt{\Sigma_{s}^{-1}} \mathrm{~d} W_{s} Q\right] \\
\log \left(\frac{\operatorname{det} \Sigma_{T}}{\operatorname{det} \Sigma_{0}}\right)^{\lambda / 2} & =\lambda \operatorname{Tr}(M)+\frac{\lambda}{2} \int_{0}^{T}(\beta-n-1) \operatorname{Tr}\left(\Sigma_{s}^{-1} Q^{T} Q\right) \mathrm{d} s+\lambda \int_{0}^{T} \operatorname{Tr}\left[\sqrt{\Sigma_{s}^{-1}} \mathrm{~d} W_{s} Q\right] \\
\left(\frac{\operatorname{det} \Sigma_{T}}{\operatorname{det} \Sigma_{0}}\right)^{-(\lambda / 2)} & =\exp \left\{-\lambda \operatorname{Tr}(M)-\frac{\lambda}{2} \int_{0}^{T}(\beta-n-1) \operatorname{Tr}\left(\Sigma_{s}^{-1} Q^{T} Q\right) \mathrm{d} s-\lambda \int_{0}^{T} \operatorname{Tr}\left[\sqrt{\Sigma_{s}^{-1}} \mathrm{~d} W_{s} Q\right]\right\} \\
e^{\lambda \int_{0}^{T} \operatorname{Tr}\left[\sqrt{v_{s}} \operatorname{ses}, s\right]} & =\left(\frac{\operatorname{det} \Sigma_{T}}{\operatorname{det} \Sigma_{0}}\right)^{\lambda / 2} \exp \{-\lambda T \operatorname{Tr}(M)\} \exp \left\{-\frac{\lambda}{2} \int_{0}^{T}(\beta-n-1) \operatorname{Tr}\left(\Sigma_{s}^{-1} Q^{T} Q\right) \mathrm{d} s\right\}
\end{aligned}
$$
by

From equation (A.2), the new measure with $\widetilde{W}$ is given

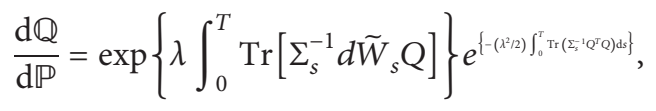

$$
\begin{aligned}
& \frac{\mathrm{d} \mathbb{Q}}{\mathrm{dP}}=\left(\frac{\operatorname{det} \Sigma_{T}}{\operatorname{det} \Sigma_{0}}\right)^{\lambda / 2} \exp \{-\lambda T \operatorname{Tr}(M)\} \exp \left\{-\frac{\lambda}{2} \int_{0}^{T}(\beta+2 \lambda-n-1) \operatorname{Tr}\left(\Sigma_{s}^{-1} Q^{T} Q\right) \mathrm{d} s\right\} \\
& \cdot \exp \left\{-\frac{\lambda^{2}}{2} \int_{0}^{T} \operatorname{Tr}\left(\Sigma_{s}^{-1} Q^{T} Q\right) \mathrm{d} s\right\} \\
& =\left(\frac{\operatorname{det} \Sigma_{T}}{\operatorname{det} \Sigma_{0}}\right)^{\lambda / 2} \exp \{-\lambda T \operatorname{Tr}(M)\} \exp \left\{\left[-\frac{\lambda}{2}(\beta+2 \lambda-n-1)-\frac{\lambda^{2}}{2}\right] \int_{0}^{T} \operatorname{Tr}\left(\Sigma_{s}^{-1} Q^{T} Q\right) \mathrm{d} s\right\} \\
& =\left(\frac{\operatorname{det} \Sigma_{T}}{\operatorname{det} \Sigma_{0}}\right)^{\lambda / 2} \exp \{-\lambda T \operatorname{Tr}(M)\} \exp \left\{-\frac{\lambda}{2}(\beta+\lambda-n-1) \int_{0}^{T} \operatorname{Tr}\left(\Sigma_{s}^{-1} Q^{T} Q\right) \mathrm{d} s\right\}, \\
& \frac{\mathrm{d} \mathbb{Q}}{\mathrm{dP}}=\left(\frac{\operatorname{det} \Sigma_{T}}{\operatorname{det} \Sigma_{0}}\right)^{\lambda / 2} \exp \{-\lambda T \operatorname{Tr}(M)\} \exp \left\{-\frac{\lambda}{2}(\beta+\lambda-n-1) \int_{0}^{T} \operatorname{Tr}\left(\Sigma_{s}^{-1} \mathrm{Q}^{T} \mathrm{Q}\right) \mathrm{d} s\right\} .
\end{aligned}
$$

The change of the probability measure is obtained.

\section{B. The Correlation Structure}

The Brownian matrices $W_{t}$ and $Z_{t}$ are correlated to give a constant correlated matrix $R \in M_{n}$, in the study by $\mathrm{Da}$ Fonseca et al. [3], which describes the correlation structure for $Z_{t}$ :

$$
Z_{t}:=W_{t} R^{T}+B_{t} \sqrt{\mathbb{q}-R R^{T}},
$$

where $\square$ is the identity matrix, $T$ is the transpose, and $B_{t}$ is an independent Brownian motion matrix from $W_{t}$. The correlation structure is a Brownian motion.

Proof. $Z_{t}$ is matrix Brownian motion if and only if $a, b \in \mathbb{R}^{n}:$

$$
\operatorname{Cov}_{t}\left(\mathrm{~d} Z_{t} a, \mathrm{~d} Z_{t} b\right)=\mathbb{E}_{t}\left[\left(\mathrm{~d} Z_{t} a\right)\left(\mathrm{d} Z_{t} b\right)^{T}\right]=a^{T} b \llbracket \mathrm{d} t .
$$

Since, 


$$
\begin{aligned}
\operatorname{Cov}_{t}\left(\mathrm{~d} Z_{t} a, \mathrm{~d} Z_{t} b\right) & =\mathbb{E}_{t}\left[\left(\mathrm{~d} W_{t} R^{T} a+d B_{t} \sqrt{\square-R R^{T} a}\right)\left(\mathrm{d} W_{t} R^{T} b+d B_{t} \sqrt{\square-R R^{T} b}\right)\right] \\
& =\operatorname{Cov}_{t}\left(\mathrm{~d} W_{t} R^{T} a, \mathrm{~d} W_{t} R^{T} b\right)+\operatorname{Cov}_{t}\left(d B_{t} \sqrt{\square-R R^{T}} a, d B_{t} \sqrt{\square-R R^{T} b}\right) \\
& =a^{T} R R^{T} b \llbracket \mathrm{d} t+a^{T}\left(\llbracket-R R^{T}\right) b \llbracket \mathrm{d} t \\
& =a^{T} b \llbracket \mathrm{d} t .
\end{aligned}
$$

\section{Brownian Motions}

In Lemma 1, we show that the following processes $X_{t}, \overline{X_{t}}, \xi_{t}$, and $\eta_{t}$ are the Brownian motions.

Proof

$$
\begin{aligned}
\operatorname{Cov}_{t}\left(\mathrm{~d} X_{t}, \mathrm{~d} X_{t}\right) & =\mathbb{E}_{t}\left[\left(\mathrm{~d} X_{t}\right)\left(\mathrm{d} X_{t}\right)^{T}\right] \\
& =\mathbb{E}_{t}\left[\frac{\operatorname{Tr}\left(\sqrt{\Sigma_{t}} \mathrm{~d} Z_{t}\right)}{\sqrt{\operatorname{Tr}\left(\Sigma_{t}\right)}} \frac{\operatorname{Tr}\left(\sqrt{\Sigma_{t}} \mathrm{~d} Z_{t}\right)}{\left.\sqrt{\operatorname{Tr}\left(\Sigma_{t}\right)}\right]}\right. \\
& =\frac{\sum_{i, j} \operatorname{Cov}_{t}\left(e_{i}^{T} \sqrt{\Sigma_{t}} \mathrm{~d} Z_{t} e_{i}, e_{j}^{T} \sqrt{\Sigma_{t}} \mathrm{~d} Z_{t} e_{j}\right)}{\sqrt{\operatorname{Tr}\left(\Sigma_{t}\right)} \sqrt{\operatorname{Tr}\left(\Sigma_{t}\right)}} \\
& =\frac{\sum_{i, j} e_{i}^{T} \sqrt{\Sigma_{t}} \operatorname{Tr}\left(e_{i} e_{j}^{T}\right) \sqrt{\Sigma_{t}} e_{j} \mathrm{~d} t}{\operatorname{Tr}\left(\Sigma_{t}\right)} \\
& =\frac{\sum_{i, j} \operatorname{Tr}\left(e_{i} e_{j}^{T}\right) e_{i}^{T} \Sigma_{t} e_{j} \mathrm{~d} t}{\operatorname{Tr}\left(\Sigma_{t}\right)} \\
& =\frac{\left.\sum_{i, j} e_{j}^{T} e_{i} e_{i}^{T}\right) e_{i}^{T} \sum_{t} e_{j} \mathrm{~d} t}{\operatorname{Tr}\left(\Sigma_{t}\right)} \\
& =\frac{\operatorname{dr} t .}{\operatorname{Tr}\left(\sum_{t}\right)} \\
& =\frac{\sum_{j} e_{j}^{T} \sum_{t} e_{j} \mathrm{~d} t}{\operatorname{Tr}\left(\sum_{t}\right)} \\
& \\
& \\
&
\end{aligned}
$$

Similarly, for Brownian motion $\overline{X_{t}}$, its proof can be obtained through the same procedures as above.

Then, we can also show that $\xi_{t}$ is a Brownian motion as follows.
Proof

$$
\begin{aligned}
\operatorname{Cov}_{t}\left(\mathrm{~d} \xi_{t}, \mathrm{~d} \xi_{t}\right) & =\mathbb{E}_{t}\left[\frac{\operatorname{Tr}\left(Q \mathrm{~d} W_{t} \sqrt{\Sigma_{t}}\right)}{\sqrt{\operatorname{Tr}\left(Q^{T} Q \Sigma_{t}\right)}} \frac{\operatorname{Tr}\left(Q \mathrm{~d} W_{t} \sqrt{\Sigma_{t}}\right)}{\sqrt{\operatorname{Tr}\left(Q^{T} Q \Sigma_{t}\right)}}\right] \\
& =\frac{\sum_{i, j} \operatorname{Cov}_{t}\left(e_{i}^{T} Q \mathrm{~d} W_{t} \sqrt{\Sigma_{t}} e_{i}, e_{j}^{T} Q \mathrm{~d} W_{t} \sqrt{\Sigma_{t}} e_{j}\right)}{\sqrt{\operatorname{Tr}\left(Q^{T} Q \Sigma_{t}\right)} \sqrt{\operatorname{Tr}\left(Q^{T} Q \Sigma_{t}\right)}} \\
& =\frac{\sum_{i, j} e_{i}^{T} \sqrt{\Sigma_{t}} \operatorname{Tr}\left(Q^{T} e_{i}, e_{j}^{T} Q\right) \sqrt{\Sigma_{t}} e_{j} \mathrm{~d} t}{\sqrt{\operatorname{Tr}\left(Q^{T} Q \Sigma_{t}\right)} \sqrt{\operatorname{Tr}\left(Q^{T} Q \Sigma_{t}\right)}} \\
& =\frac{\sum_{i, j} \operatorname{Tr}\left(Q^{T} Q e_{i} e_{j}^{T}\right) e_{i}^{T} \Sigma_{t} e_{j} \mathrm{~d} t}{\operatorname{Tr}\left(Q^{T} Q \Sigma_{t}\right)} \\
& =\frac{\sum_{i, j} e_{j}^{T} Q^{T} Q e_{i} e_{i}^{T} \Sigma_{t} e_{j} \mathrm{~d} t}{\operatorname{Tr}\left(Q^{T} Q \Sigma_{t}\right)} \\
& =\frac{\operatorname{d} t .}{\sum_{i, j} e_{j}^{T} Q^{T} Q \Sigma_{t} e_{j} \mathrm{~d} t} \\
& \operatorname{Tr}\left(Q^{T} Q \Sigma_{t}\right) \\
& \frac{\operatorname{Tr}\left(Q^{T} Q \Sigma_{t}\right) \mathrm{d} t}{\left.\Sigma_{t}\right)}
\end{aligned}
$$

The same procedures can be followed to show that $\eta_{t}$ is a Brownian motion.

\section{Characteristic Function of the Double Wishart Model}

Let $D$ be symmetric matrix, and the conditional characteristic function of the double Wishart $\Sigma_{t}$ and $\bar{\Sigma}_{t}$ is given by 


$$
\begin{aligned}
\tilde{\phi}_{\Sigma_{t}, \bar{\Sigma}_{t}}^{D_{1} D_{2}} & =\mathbb{E}\left[\exp \left\{i \operatorname{Tr}\left[D_{1} \Sigma_{t}+D_{2} \bar{\Sigma}_{t}\right]\right\}\right] \\
& =\exp \left\{\operatorname{Tr}\left[A_{1}(\tau) \Sigma_{t}+A_{2}(\tau) \bar{\Sigma}_{t}\right]+C(\tau)\right\},
\end{aligned}
$$

Proof. Obtaining expressions of $A_{1}(\tau), A_{2}(\tau)$, and $C(\tau)$, through the Riccati equations,

where $A_{1}(\tau), A_{2}(\tau) \in M_{n}$, and $C \in \mathbb{C}$ verify the following dynamics.

$$
\begin{aligned}
& \left\{\begin{array}{l}
\frac{\mathrm{d} A_{1}(\tau)}{\mathrm{d} \tau}=A_{1}(\tau) M+M^{T} A_{1}(\tau)+2 A_{1}(\tau) Q^{T} Q A_{1}(\tau), \\
A_{1}(0)=i D_{1},
\end{array}\right. \\
& \left\{\begin{array}{l}
\frac{\mathrm{d} A_{2}(\tau)}{\mathrm{d} \tau}=A_{2}(\tau) \bar{M}+\bar{M}^{T} A_{2}(\tau)+2 A_{2}(\tau) \bar{Q}^{T} \bar{Q} A_{2}(\tau), \\
A_{2}(0)=i D_{2},
\end{array}\right. \\
& \left\{\begin{array}{l}
\frac{\mathrm{d} C(\tau)}{\mathrm{d} \tau}=\beta \operatorname{Tr}\left[Q^{T} Q A_{1}(\tau)\right]+\bar{\beta} \operatorname{Tr}\left[\bar{Q}^{T} \bar{Q} A_{2}(\tau)\right], \\
C(0)=0 .
\end{array}\right.
\end{aligned}
$$

Now,

$$
\begin{aligned}
& A_{1}(\tau)=h_{1}^{-1}(\tau) h_{2}(\tau), \\
& \frac{\mathrm{d}}{\mathrm{d} \tau}\left(h_{2}(\tau) h_{1}(\tau)\right)=\left(\begin{array}{ll}
h_{2}(\tau) & \left.h_{1}(\tau)\right)
\end{array}\right)\left(\begin{array}{cc}
M & -2 Q^{T} Q \\
0 & -M^{T}
\end{array}\right), \\
& A_{2}(\tau)=i_{1}^{-1}(\tau) i_{2}(\tau), \\
& \frac{\mathrm{d}}{\mathrm{d} \tau}\left(i_{2}(\tau) i_{1}(\tau)\right)=\left(\begin{array}{lll}
i_{2}(\tau) & i_{1}(\tau)
\end{array}\right)\left(\begin{array}{cc}
\bar{M} & -2 \overline{\mathrm{Q}}^{T} \overline{\mathrm{Q}} \\
0 & -\bar{M}^{T}
\end{array}\right) .
\end{aligned}
$$

We have,

$$
\begin{aligned}
& \left(\begin{array}{ll}
h_{2}(\tau) & h_{1}(\tau)
\end{array}\right)=\left(\begin{array}{ll}
i D_{1} & \mathbb{q}_{n}
\end{array}\right)\left(\begin{array}{ll}
A_{1}^{11}(\tau) & A_{1}^{12}(\tau) \\
A_{1}^{21}(\tau) & A_{1}^{22}(\tau)
\end{array}\right), \\
& \left(\begin{array}{ll}
i_{2}(\tau) & i_{1}(\tau)
\end{array}\right)=\left(\begin{array}{ll}
i D_{2} & \mathbb{q}_{n}
\end{array}\right)\left(\begin{array}{ll}
A_{2}^{11}(\tau) & A_{2}^{12}(\tau) \\
A_{2}^{21}(\tau) & A_{2}^{22}(\tau)
\end{array}\right), \\
& \left(h_{2}(\tau) h_{1}(\tau)\right)=\left(i D_{1} A_{1}^{11}(\tau)+A_{1}^{21}(\tau) i D_{1} A_{1}^{12}(\tau)+A_{1}^{22}(\tau)\right) \text {, } \\
& \left(i_{2}(\tau) i_{1}(\tau)\right)=\left(i D_{2} A_{2}^{11}(\tau)+A_{2}^{21}(\tau) i D_{2} A_{2}^{12}(\tau)+A_{2}^{22}(\tau)\right),
\end{aligned}
$$

where

$$
\begin{aligned}
& A_{1}(\tau)=h_{1}^{-1}(\tau) h_{2}(\tau), \\
& h_{1}(\tau)=i D_{1} A_{1}^{12}(\tau)+A_{1}^{22}(\tau), \\
& h_{2}(\tau)=i D_{1} A_{1}^{11}(\tau)+A_{1}^{21}(\tau), \\
& A_{1}(\tau)=\left(i D_{1} A_{1}^{12}(\tau)+A_{1}^{22}(\tau)\right)^{-1}\left(i D_{1} A_{1}^{11}(\tau)+A_{1}^{21}(\tau)\right), \\
& A_{2}(\tau)=i_{1}^{-1}(\tau) i_{2}(\tau), \\
& i_{1}(\tau)=i D_{2} A_{2}^{12}(\tau)+A_{2}^{22}(\tau), \\
& i_{2}(\tau)=i D_{2} A_{2}^{11}(\tau)+A_{2}^{21}(\tau), \\
& A_{2}(\tau)=\left(i D_{2} A_{2}^{12}(\tau)+A_{2}^{22}(\tau)\right)^{-1}\left(i D_{2} A_{2}^{11}(\tau)+A_{2}^{21}(\tau)\right) .
\end{aligned}
$$

\section{Data Availability}

The data used to support the findings of this study are available from the corresponding author upon request.

\section{Conflicts of Interest}

The authors declare that they have no conflicts of interest.

\section{Acknowledgments}

The authors extend their gratitude to the Pan African University Institute for Basic sciences, Technology and Innovation (Pausti) at Jomo Kenyatta University. 


\section{References}

[1] F. Black and M. Scholes, "The pricing of options and corporate liabilities," Journal of Political Economy, vol. 81, no. 3, pp. 637-654, 1973.

[2] S. L. Heston, "A closed-form solution for options with stochastic volatility with applications to bond and currency options," Review of Financial Studies, vol. 6, no. 2, pp. 327-343, 1993.

[3] J. Da Fonseca, M. Grasselli, and C. Tebaldi, "A multifactor volatility Heston model," Quantitative Finance, vol. 8, no. 6, pp. 591-604, 2008.

[4] P. Christoffersen, S. Heston, and K. Jacobs, "The shape and term structure of the index option smirk: why multifactor stochastic volatility models work so well," Management Science, vol. 55, no. 12, pp. 1914-1932, 2009.

[5] A. Ahdida and A. Alfonsi, "Exact and high-order discretization schemes for Wishart processes and their affine extensions," The Annals of Applied Probability, vol. 23, no. 3, pp. 1025-1073, 2013.

[6] C. Kang, W. Kang, and J. M. Lee, "Exact simulation of the Wishart multidimensional stochastic volatility model," $O p$ erations Research, vol. 65, no. 5, pp. 1190-1206, 2017.

[7] G. La Bua and D. Marazzina, "Calibration and advanced simulation schemes for the Wishart stochastic volatility model," Quantitative Finance, vol. 19, no. 6, pp. 997-1016, 2019.

[8] C. Gouriéroux, "Continuous time Wishart process for stochastic risk," Econometric Reviews, vol. 25, no. 2-3, pp. 177-217, 2006.

[9] A. Benabid, H. Bensusan, and N. El Karoui, Wishart stochastic volatility: asymptotic smile and numerical framework, 2008.

[10] C. Kang and W. Kang, "Exact simulation of wishart multidimensional stochastic volatility model," 2013, https://arxiv. org/pdf/1309.0557.

[11] M.-F. Bru, "Wishart processes," Journal of Theoretical Probability, vol. 4, no. 4, pp. 725-751, 1991.

[12] M. Katori, Bessel Processes, Schramm-Loewner Evolution, and the Dyson Model, Vol. 11, Springer, Berlin, Germany, 2016.

[13] A. Alfonsi, Affine Diffusions and Related Processes: Simulation, Theory and Applications, Vol. 6, Springer, Berlin, Germany, 2015.

[14] C. Gourieroux and R. Sufana, "Derivative pricing with Wishart multivariate stochastic volatility: application to credit risk," SSRN Electronic Journal, pp. 1-44, 2004.

[15] D. Duffie, J. Pan, and K. Singleton, "Transform analysis and asset pricing for affine jump-diffusions," Econometrica, vol. 68, no. 6, pp. 1343-1376, 2000.

[16] P. Carr and D. Madan, "Option valuation using the fast fourier transform," The Journal of Computational Finance, vol. 2, no. 4, pp. 61-73, 1999.

[17] J. P. Fouque, G. Papanicolaou, R. Sircar, and K. Solna, "Singular perturbations in option pricing," SIAM Journal on Applied Mathematics, vol. 63, no. 5, pp. 1648-1665, 2003.

[18] T. Björk, Arbitrage Theory in Continuous Time, Oxford University Press, Oxford, UK, 2009.

[19] S. E. Shreve, Stochastic Calculus for Finance II: ContinuousTime Models, Vol. 11, Springer Science \& Business Media, Berlin, Germany, 2004.

[20] D. Filipovic and E. Mayerhofer, "Affine diffusion processes: theory and applications," Advanced Financial Modelling, vol. 8, pp. 1-40, 2009. 